\title{
A Dynamical Systems Analysis of the Data Assimilation Linked Ecosystem Carbon (DALEC) Models
}

\author{
Anna M. Chuter, Philip J. Aston, ${ }^{\text {a) }}$ Anne C. Skeldon, and lan Roulstone \\ Department of Mathematics, University of Surrey, Guildford, Surrey GU2 7XH, \\ $U K$
}

(Dated: 8 December 2014)

Changes in our climate and environment make it ever more important to understand the processes involved in Earth systems, such as the carbon cycle. There are many models that attempt to describe and predict the behaviour of carbon stocks and stores but, despite their complexity, significant uncertainties remain. We consider the qualitative behaviour of one of the simplest carbon cycle models, the Data Assimilation Linked Ecosystem Carbon (DALEC) model, which is a simple vegetation model of processes involved in the carbon cycle of forests, and consider in detail the dynamical structure of the model. Our analysis shows that the dynamics of both evergreen and deciduous forests in DALEC are dependent on a few key parameters and it is possible to find a limit point where there is stable sustainable behaviour on one side but unsustainable conditions on the other side. The fact that typical parameter values reside close to this limit point highlights the difficulty of predicting even the correct trend without sufficient data and has implications for the use of data assimilation methods.

PACS numbers: 05.10.-a, 02.30.Oz, 89.60.-k

Keywords: Carbon cycle, evergreen and deciduous forests, DALEC models, data assimilation, nonlinear dynamics.

\footnotetext{
a)Electronic mail: P.Aston@surrey.ac.uk
} 
One important aspect of understanding global climate change is to develop computational models that enable us to predict the extent of global warming under different scenarios. One part of these computational models is to understand the role of forests in determining atmospheric carbon and to this end, a number of sophisticated models to look at carbon balances in forests have been developed.

With complicated models it can be difficult to develop an intuition for what causes them to behave in the way they do and therefore what impact any intervention will have. In this paper, we have therefore looked at a relatively simple model of the carbon cycle in forests to develop an understanding of how different processes interact. This model, known as the DALEC model, is important because it contains the same fundamental processes as many more complicated models and we expect the same underlying dynamical behaviour to occur in more complicated models.

We show that the DALEC model contains a transition point where, for parameters on one side forests thrive, but on the other they die. Analysing data from two forests we find that they sit near the transition point, and we hypothesize that this is a natural consequence of a tree's need to optimize its resources. Such transition points are important because, in a changing world, we expect parameter values to drift. Drifting across a transition point can have serious consequences and results in "tipping" from one kind of behaviour to another. For example, a shift in the amount of rainfall could induce tipping from a sustainable forest to a forests that dies. The presence of such transition points also has important consequences for prediction. Models often contain many parameters that need to be determined, usually by fitting to data. Different algorithms used to fit models to data typically lead to slightly different values for parameters. Away from transition points for non-chaotic systems, this usually means that there is some small uncertainty in the parameter values that leads to a small uncertainty in prediction. However, near transitions points, small differences in parameter values can lead to very different predictions. 


\section{INTRODUCTION}

The continual increase in computational power brings with it the ability to perform evermore detailed studies of the Earth system and its components. Such studies help underpin the debate on climate change and global warming. Stocks and fluxes of carbon are an important component of this debate and, within this, the role of terrestrial vegetation and its influence on climate. Terrestrial vegetation is responsible for the removal of a large percentage of the carbon dioxide from the atmosphere every year. However, with increasing carbon dioxide levels and the consequent global warming, terrestrial vegetation may absorb less carbon dioxide or may even contribute to a further increase in atmospheric carbon dioxide by dying and decomposing ${ }^{1}$.

Various sophisticated models are used in the analysis of the carbon cycle including

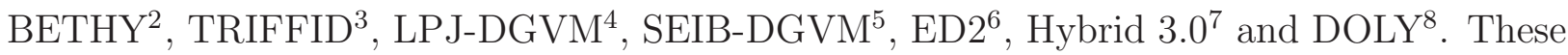
are called Dynamic Global Vegetation Models (DGVMs) and involve many physiological processes of the terrestrial carbon cycle through both empirical and process based methods. They are typically quite complex and are driven by climate data. They often distinguish between a large number of plant functional types. For example, BETHY allows different photosynthesis models to be used and has vegetation maps rather than plant functional types. LPJ-DGVM allows for ten different plant types, whereas TRIFFID uses five, and SEIB even simulates the local interaction among individual trees. Photosynthesis, evapotranspiration and soil water dynamics are modelled, using meteorological data on various time scales and considering different layers of soil.

DGVMs are used both for determining carbon stocks and stores and also to simulate the effects of future climate change on natural vegetation by combining them with General Circulation Models (GCMs). For example BETHY has been coupled with a transport model, TM2 ${ }^{9}$, for the CCDAS (Carbon Cycle Data Assimilation System) consortium. TRIFFID has been coupled to HADCM3 ${ }^{3}$ and JULES $^{10}$ and in a study by Schaphoff ${ }^{11}$, LPJ-DGVM was coupled to five different GCMs to compare land biosphere carbon uptake between the GCMs under five different simulations of climate change driven by a common emission scenario of $\mathrm{CO}_{2}$ increase.

As our knowledge of processes in the carbon cycle grow it is tempting to extend existing models making them ever more complex. However, the underlying dynamics of the carbon 
cycle are not well understood, not least because the values of many of the parameters that appear in the DGVMs are not known with confidence. In some cases parameters are difficult to measure and in others the data that would be needed to constrain the parameters is very limited. Uncertainties in observations and parameters influence the outcome of a simulation of a complex system, such as a GCM, as a consequence of changes in the dynamics of the core model. Indeed, it has been suggested that as our understanding develops of the many factors that play a role, such as the concentration of greenhouse gases, uncertainty in mechanisms and their model depiction, the weighting of global climate models and uncertainty in projected regional changes, that estimates of complex climate processes will include more uncertainty in their predictions rather than less ${ }^{12}$.

Various projects have been designed to use techniques from data assimilation in an attempt to constrain the values of the parameters by 'confronting the models with data' 13,14 . The majority of modern data assimilation schemes use the forward model as part of the algorithm, and it is vital to understand the nature of the system dynamics if one is to employ the data assimilation methods with any confidence. For example, variational data assimilation techniques are based on assumptions of quasi-linearity and the use of Gaussian statistics. The presence of multiple timescales, either in the frequency of observations or in the dynamics of the forward model, is a feature that presents challenges to data assimilation schemes. Understanding the dynamics of carbon cycle models brings insight as to whether they are amenable to data assimilation methods given the various parameter sensitivities and the intrinsic separation of timescales, from days (for foliar carbon) to millennia (for soil organic matter).

In order to begin studying the issues that arise in more complex systems we study a relatively simple process model which is typical of that which underpins many GCMs. This is a strategy not dissimilar to that found in other areas of environmental prediction, for example the Lorenz 1963 equations $^{15}$, which were used to study thermal convection in a very simple setting and which brought to light fundamental properties of dynamical systems. We therefore focus on the Data Assimilation Linked Ecosystem Carbon (DALEC) model ${ }^{16}$. DALEC is a dynamic vegetation model which simulates the carbon cycle of forests and two variants are considered here, one for an evergreen forest and one for a deciduous forest. It was especially created for data assimilation and specifically designed for calibration and testing against eddy flux data. DALEC is a simple box model of carbon pools connected via 
fluxes running at a daily time-step, driven by daily climate data such as temperature, irradiance and atmospheric carbon concentration, that aims to capture the essential dynamics of the carbon cycle. Like many other DGVMS, such as ED2 ${ }^{6}$, Hybrid $3.0^{7}$ and DOLY ${ }^{8}$, the modelling of photosynthesis in DALEC is based on the Farquhar photosynthesis model ${ }^{17}$.

DALEC's simplicity limits its application to detailed studies of terrestrial carbon systems, but in turn it facilitates close mathematical scrutiny. We shall show how the generic behaviour of this model is controlled by various parameters. The techniques we develop from dynamical systems theory may be applied to more complex models, such as BETHY or TRIFFID, but it is surely appropriate to describe and test the basic techniques on a model that permits explicit and detailed analysis.

We formulate the process model for DALEC as a dynamical system, iterative in time, and we study the model using dynamical systems methods, employing both analytical and numerical approaches. The work was motivated by a masters thesis by $\operatorname{Ilett}^{18}$, and our analysis sets Ilett's work in a broader context. We show how the model predicts different dynamical behaviour for different parameter regions predicting, for the evergreen version of DALEC, that if the amount of carbon allocated to foliage is not in balance with the daily turnover rate of foliage then widespread mortality of the forest will result.

In Section II the DALEC evergreen (DALEC EV) and deciduous (DALEC DE) models are formulated as daily maps. The behaviour of the evergreen model is studied in Section III by finding fixed points of the annual map. The effect of smoothing the highly variable daily driver data is also considered. Similar methods are applied to the DALEC deciduous (DALEC DE) model in Section IV. Finally, in Section V, an interpretation of the results and a discussion of their consequences are presented.

\section{MODEL DESCRIPTION, DATA AND METHODS}

\section{A. Model Description and Data}

The DALEC EV model ${ }^{16}$ describes the time evolution of five carbon pools, namely foliage $\left(C_{f}\right)$, woody stems and coarse roots $\left(C_{w}\right)$, fine roots $\left(C_{r}\right)$, fresh leaf and fine root litter $\left(C_{l i t}\right)$ and soil organic matter and coarse woody debris $\left(C_{\text {som }}\right)$, together with one pseudo-pool, Gross Primary Production (GPP). The carbon cycle of an evergreen forest according to 


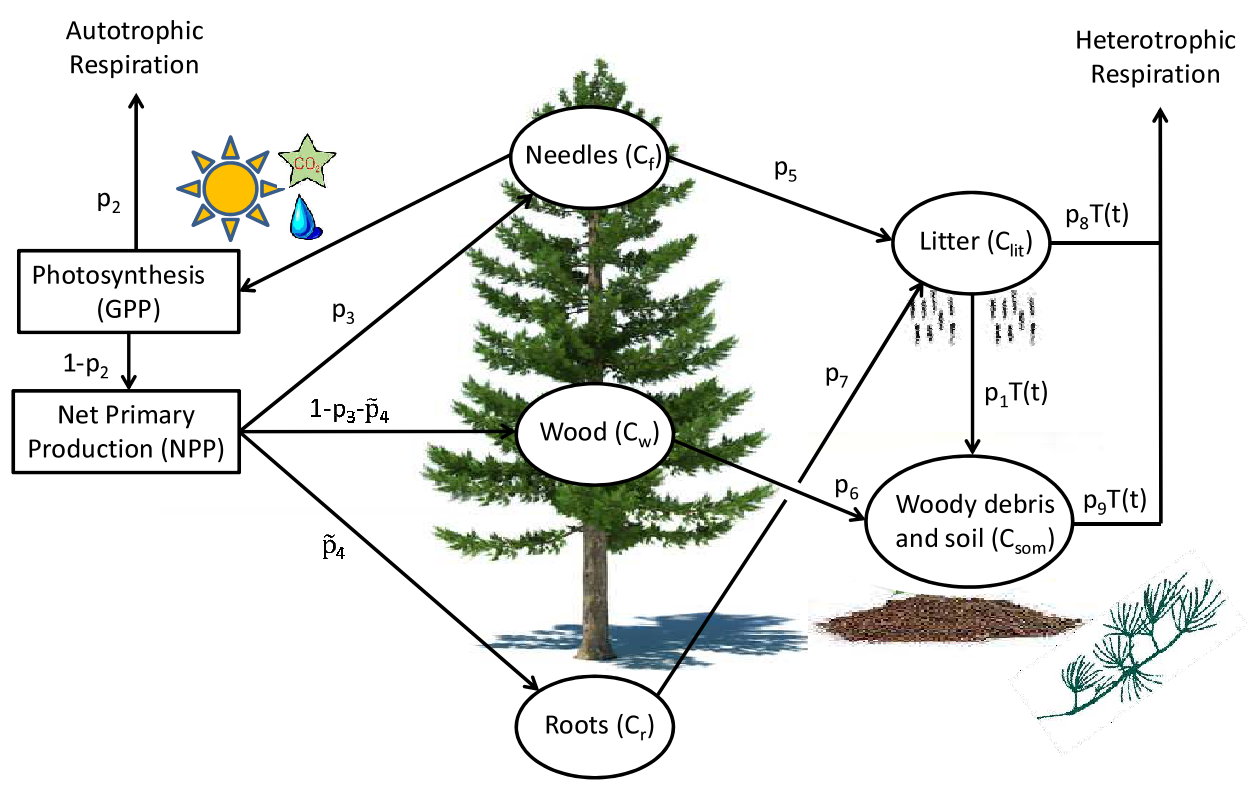

FIG. 1. A schematic overview of the carbon cycle of an evergreen forest according to DALEC EV.

DALEC is shown in Fig. 1. The pools are connected by arrows, which represent allocation and production fluxes. Sunlight, water and $\mathrm{CO}_{2}$ are needed for photosynthesis which takes place in the needles. The photosynthate is stored in the GPP, which distributes the available carbon to the $C_{f}, C_{w}$ and $C_{r}$ pools. During photosynthesis carbon is also respired into the atmosphere by the tree, which is called autotrophic respiration $\left(R_{a}\right)$. Decomposition of litter in and on the soil results in heterotrophic respiration $\left(R_{h}\right)$.

For our purposes, we express the DALEC EV model equations ${ }^{19}$ as a discrete dynamical system in the form of daily maps, specifically:

$$
\begin{aligned}
C_{f}(t+1) & =\left(1-p_{5}\right) C_{f}(t)+p_{3}\left(1-p_{2}\right) G P P\left(C_{f}(t), t\right), \\
C_{r}(t+1) & =\left(1-p_{7}\right) C_{r}(t)+\tilde{p}_{4}\left(1-p_{2}\right) G P P\left(C_{f}(t), t\right), \\
C_{w}(t+1) & =\left(1-p_{6}\right) C_{w}(t)+\left(1-p_{3}-\tilde{p}_{4}\right)\left(1-p_{2}\right) G P P\left(C_{f}(t), t\right), \\
C_{l i t}(t+1) & =\left(1-\left(p_{8}+p_{1}\right) T(t)\right) C_{l i t}(t)+p_{5} C_{f}(t)+p_{7} C_{r}(t), \\
C_{\text {som }}(t+1) & =\left(1-p_{9} T(t)\right) C_{\text {som }}(t)+p_{6} C_{w}(t)+p_{1} T(t) C_{l i t}(t),
\end{aligned}
$$

where $t$ is time in days and $T(t)=0.5 \exp \left(p_{10} T_{m}(t)\right)$ and $T_{m}(t)$ is the mean daily air temperature, which is the mean of the maximum and minimum daily temperatures. The term $T(t)$ models the fact that the decomposition of leaf litter and of soil and organic matter 
by fungi and bacteria is strongly dependent on temperature, with decomposition occurring more rapidly at higher temperatures. For a description of the parameters and the values used see Appendix A.

The Net Primary Production (NPP) is the net carbon flux from the atmosphere into the trees and is defined by

$$
N P P\left(C_{f}(t), t\right)=G P P\left(C_{f}(t), t\right)-R_{a}=\left(1-p_{2}\right) G P P\left(C_{f}(t), t\right)
$$

where $R_{a}=p_{2} G P P\left(C_{f}(t), t\right)$ is the autotrophic respiration. We note that in equations (1)(3), it is this NPP that is allocated to the foliar, roots and wood pool with corresponding fractions $p_{3}, \tilde{p}_{4}$ and $1-p_{3}-\tilde{p}_{4}$. We note that the definition of the DALEC model in ${ }^{19}$ used a slightly different definition of these constants, and our parameter $\tilde{p}_{4}$ is defined in terms of the original parameter $p_{4}$ by

$$
\tilde{p}_{4}=p_{4}\left(1-p_{3}\right)
$$

However, our notation is consistent with that used $\mathrm{in}^{16}$, where the parameters $t_{3}$ and $t_{4}$ correspond to our parameters $p_{3}$ and $\tilde{p}_{4}$.

Typical values for the initial conditions for the five pools used by participants of the REgional FLux Estimation eXperiment (REFLEX) ${ }^{19}$ are $C_{f}(0)=150 \mathrm{gC} \mathrm{m}^{-2}, C_{r}(0)=160$ $\mathrm{gC} \mathrm{m}^{-2}, C_{\text {lit }}(0)=60 \mathrm{gC} \mathrm{m}^{-2}, C_{w}(0)=9200 \mathrm{gC} \mathrm{m}^{-2}$ and $C_{\text {som }}(0)=11000 \mathrm{gC} \mathrm{m}^{-2}$, where day zero is taken to be $21^{\text {st }}$ December, the shortest day of the year.

DALEC deciduous (DALEC DE) is another version of the DALEC model, see Fig. 2, which includes the same five carbon pools as in the evergreen model, together with an additional labile pool. This pool stores carbon during the winter once the leaves have dropped and is then depleted during the spring and summer when the leaves grow again. As for DALEC EV, we express the DALEC DE model equations ${ }^{19}$ in the form of daily maps, specifically:

$$
\begin{aligned}
C_{f}(t+1)= & \left(1-p_{5}\left(p_{14}+\left(1-p_{14}\right) T(t)\right) m_{t f}\right) C_{f}(t) \\
& +\min \left(p_{17}-C_{f}(t), p_{3}\left(1-p_{2}\right) G P P\left(C_{f}(t), t\right)\right) m_{t l} \\
& +p_{15}\left(1-p_{16}\right) C_{l a b}(t) T(t) m_{t l}, \\
C_{l a b}(t+1)= & \left(1-p_{15} T(t) m_{t l}\right) C_{l a b}(t)+p_{5}\left(1-p_{14}\right)\left(1-p_{16}\right) T(t) m_{t f} C_{f}(t), \\
C_{r}(t+1)= & \left(1-p_{7}\right) C_{r}(t)+p_{4}\left(1-p_{2}\right) G P P\left(C_{f}(t), t\right) \\
& -p_{4} \min \left(p_{17}-C_{f}(t), p_{3}\left(1-p_{2}\right) G P P\left(C_{f}(t), t\right)\right) m_{t l},
\end{aligned}
$$




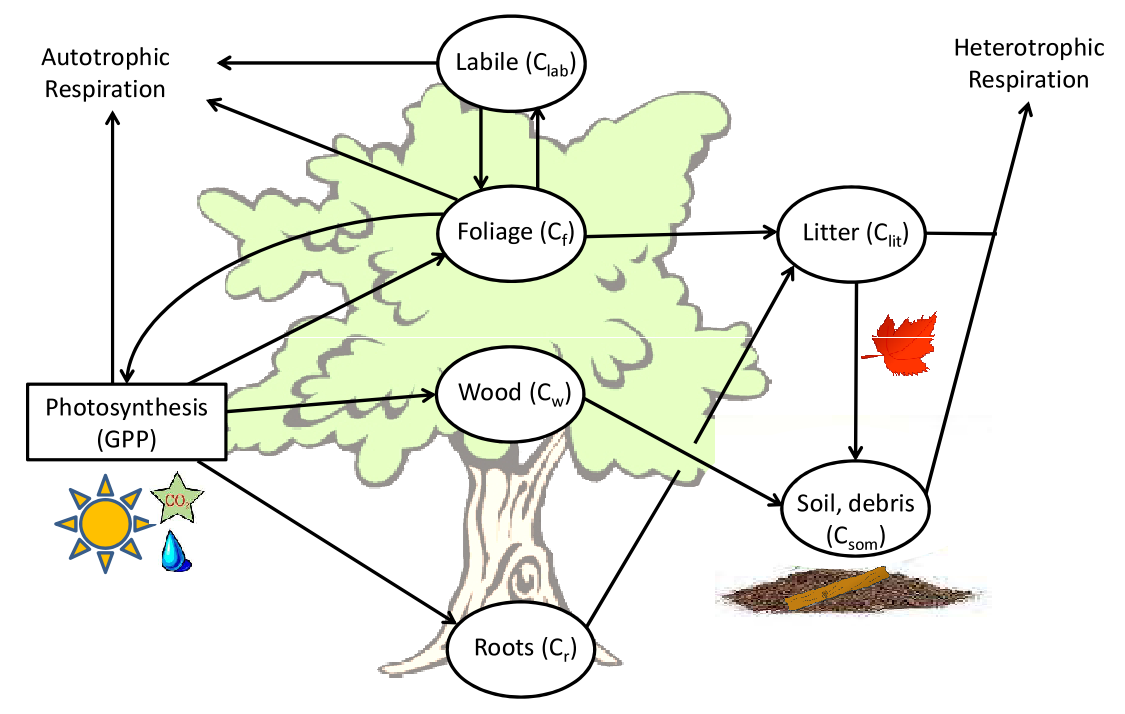

FIG. 2. A schematic overview of the carbon cycle of a deciduous forest according to DALEC DE.

$$
\begin{aligned}
C_{w}(t+1)= & \left(1-p_{6}\right) C_{w}(t)+\left(1-p_{4}\right)\left(1-p_{2}\right) G P P\left(C_{f}(t), t\right) \\
& -\left(1-p_{4}\right) \min \left(p_{17}-C_{f}(t), p_{3}\left(1-p_{2}\right) G P P\left(C_{f}(t), t\right)\right) m_{t l}, \\
C_{l i t}(t+1)= & \left(1-\left(p_{1}+p_{8}\right) T(t)\right) C_{l i t}(t)+p_{5} p_{14} m_{t f} C_{f}(t)+p_{7} C_{r}(t), \\
C_{\text {som }}(t+1)= & \left(1-p_{9} T(t)\right) C_{\text {som }}(t)+p_{6} C_{w}(t)+p_{1} T(t) C_{l i t}(t),
\end{aligned}
$$

where $t$ is time in days and $T(t)=0.5 \exp \left(p_{10} T_{m}(t)\right)$ is the temperature sensitive rate parameter. $T_{m}(t)$ is the mean daily air temperature, which is the mean of the maximum and minimum daily temperatures. There are 17 parameters in DALEC DE; for a description and the values used see Appendix A. There are two switches in the model, $m_{t f}$ and $m_{t l}$, which have values of either zero or one. The variable $m_{t f}$ switches the turnover of foliar carbon on (in the autumn, so that the trees drop leaves) or off (during the summer, when the trees do not drop leaves and in the spring, when the leaves are growing) and $m_{t l}$ switches the turnover of labile carbon on (used to kickstart the foliar carbon in the spring) or off (in the summer, when it has been depleted). The algorithm for determining the values of these switches is given in the ${ }^{20}$. Typical initial conditions for the five pools are: $C_{f}(0)=0 \mathrm{~g} \mathrm{Cm}^{-2}$, $C_{l a b}(0)=100 g C m^{-2}, C_{r}(0)=5 g C m^{-2}, C_{l i t}(0)=5 g C m^{-2}, C_{w}(0)=5 g C m^{-2}$ and $C_{\text {som }}(0)=9900 \mathrm{~g} C \mathrm{~m}^{-2}$. Day zero is again taken to be $21^{\text {st }}$ December, where the parameter values and the values of the initial conditions for the carbon pools are taken from ${ }^{14}$. 
The GPP for both the evergreen and the deciduous versions represents a daily accumulation of carbon produced by photosynthesis. It takes the following form:

$$
G P P\left(C_{f}(t), t\right)=\frac{E_{0}\left(C_{f}(t)\right) I(t) g_{c}(t)\left(C_{a}(t)-C_{i}\left(C_{f}(t), t\right)\right)}{E_{0}\left(C_{f}(t)\right) I(t)+g_{c}(t)\left(C_{a}(t)-C_{i}\left(C_{f}(t), t\right)\right)}\left(a_{2} s(t)+a_{5}\right) .
$$

The functions in the GPP are collectively called the Aggregated Canopy Model (ACM). A description of the various functions in the GPP can be found in Appendix B. The ACM is a daily time step model that estimates GPP as a function of Leaf Area Index (LAI), foliar nitrogen, total daily irradiance, maximum and minimum daily temperature, day length, atmospheric $\mathrm{CO}_{2}$ concentration, soil-plant water potential and total soil-plant hydraulic resistance. The LAI is the projected leaf area (in $m^{2}$ ) per ground area (also in $m^{2}$ ). The $\mathrm{ACM}^{21}$ has been derived from the Soil-Plant-Atmosphere model (SPA) by ${ }^{22}$. The SPA model is a process-based multi-layer (fine-scale) model simulating ecosystem photosynthesis and water balance at a 30 minute time step and for multiple canopy and soil layers. It uses the Farquhar model of leaf-level photosynthesis and the Penman-Monteith equation to predict evapotranspiration ${ }^{23}$. The equations for the ACM were derived from the SPA using cumulative or average values of the most sensitive driving variables. Then the ACM was calibrated so that the estimates of the GPP were similar to those of the SPA model across a wide range of the driving variables. The only parameter in the ACM which needs calibrating per site is the nitrogen use efficiency parameter, $p_{11}$. This parameter determines the maximum rate of carboxylation per gramme of foliar nitrogen.

In the original Fortran code for DALEC EV and DALEC DE provided by REFLEX ${ }^{19}$, the LAI is set to $\max \left(0.1, C_{f} / \mathrm{lma}\right)$, where lma is the leaf mass per area, which prevents it from going below 0.1. This is done in order to prevent $C_{f}$ from ever reaching zero. We note that the GPP function is undefined, as written, when $C_{f}=0$ since both the numerator and the denominator are then zero. However, we claim that there is a well-defined limit as $C_{f} \rightarrow 0$, and more precisely, that $\lim _{C_{f} \rightarrow 0} G P P\left(C_{f}, t\right)=0$. To see this, we note that for small $C_{f}, E_{0}=O\left(C_{f}^{2}\right)$ and $C_{a}-C_{i}=O\left(C_{f}\right)$. Therefore, the numerator of $(12)$ is $O\left(C_{f}^{3}\right)$ and the denominator is $O\left(C_{f}\right)$. Cancelling a factor of $C_{f}$ in the numerator and the denominator then shows that $G P P\left(C_{f}, t\right)=O\left(C_{f}^{2}\right)$ and so $G P P(0, t)=0$ as claimed. This implies that when there is no foliar carbon, there is no photosynthesis, which seems entirely reasonable. Setting the LAI to $\max \left(0.1, C_{f} / \mathrm{lma}\right)$ seems like an artificial constraint and since the model remains well-defined for $C_{f}=0$ we change the definition of the LAI to be LAI $=C_{f} / \operatorname{lma}$. 
The implication of removing this constraint is discussed in further detail below.

In order to compute the GPP, daily drivers of maximum and minimum temperature (from which the temperature range and the mean daily temperature are derived) and solar irradiance are needed. In the REFLEX project ${ }^{19}$, the atmospheric carbon $C_{a}$ increased by a small amount each year, but we have kept this value constant, although later on we will consider the effect of changing the value of this constant. The other parameters in the ACM, for example soil-plant water potential and foliar nitrogen, can be set to appropriate fixed values for the vegetation in question. Latitude also plays an important role, as it influences day length and therefore the amount of photosynthate produced.

In the DALEC model the GPP depends on the foliar carbon, $C_{f}$, via two functions, namely $E_{0}(t)$ (canopy level quantum yield) and $C_{i}(t)\left(\mathrm{CO}_{2}\right.$ concentration at site of carboxylation). This is because foliage produces the photosynthate, which none of the other carbon pools do. Therefore the foliar carbon pool is crucial in determining the amount of carbon the other pools receive and we will show that it is this pool that determines the fate of the forest.

From the model it is also possible to evaluate the net ecosystem exchange (NEE), which is the net carbon uptake or loss by the ecosystem. NEE can be calculated as follows:

$$
N E E(t)=R_{a}(t)+R_{h}(t)-G P P\left(C_{f}(t), t\right)
$$

where

$$
R_{a}(t)=p_{2} G P P\left(C_{f}(t), t\right)
$$

for DALEC EV and

$$
R_{a}(t)=p_{2} G P P\left(C_{f}(t), t\right)+p_{5}\left(1-p_{14}\right) p_{16} T(t) m_{t f} C_{f}(t)+p_{15} p_{16} T(t) m_{t l} C_{l a b}
$$

for DALEC DE, and

$$
R_{h}(t)=R_{h_{1}}(t)+R_{h_{2}}(t)=p_{8} T(t) C_{l i t}(t)+p_{9} T(t) C_{\text {som }}(t)
$$

Note that we have ignored the dependence on the various carbon pools for NEE and the respiration terms. If the forest is a carbon sink, the NEE will be negative and it will be a carbon source if NEE is positive.

In order to run the DALEC models, estimates of the parameters and values for the driver data are needed. We have used realistic data both for parameters and climate data from a European flux site, which was provided for the REFLEX project ${ }^{19}$. The purpose of this project was to compare the strengths and weaknesses of various data assimilation methods 
for estimating parameters and predicting carbon fluxes using DALEC, based on three years of driver data. However, in our analysis, the behaviour of the models is studied over a longer period of time, so driver data for this was created by averaging the three years of available data to produce mean daily data, which varies on a daily basis in a realistic manner, but is the same each year. Although daily averaging introduces some smoothing, the drivers remain highly variable. Since taking an extreme and smoothing the data to remove all but the annual variation, as discussed in Section V, has little impact, the small amount of smoothing introduced by taking average daily values over a three year period data should have a negligible effect. The time scale has been shifted ten days back, so that $t=0$ occurs on the $21^{\text {st }}$ December, the shortest day. This was done in order to ensure that the daylength function is even around zero (which simplifies the analysis). The last ten days of data were moved up to the beginning of the file to provide data for the first ten days. For simplicity, we ignore leap years, so that every year has 365 days.

Although we have used periodic drivers (for temperature and irradiance data) in this analysis, in reality they are not periodic. This lack of periodicity has two components: firstly, the solar luminosity fluctuates due to changing bright and dark structures (such as sunspots) on the solar disk ${ }^{24}$. This introduces an approximate $0.1 \%$ variation in solar irradiance reaching the Earth's upper atmosphere over and above the periodic variation due to time of year. Secondly, and much more significantly, local weather patterns lead to large variations in solar irradiance and temperature at the Earth's surface. For example, for the temperate forest in the Northern hemisphere of interest here, although there is an annual pattern of higher levels of irradiance and higher temperatures in the summer as compared with the winter, on the same day in successive years the irradiance and temperature can vary by a factor of two. However, if we assume that the drivers are periodic but with some natural variability then this lack of exact periodicity effectively corresponds to a small amount of noise in the system which would not change the overall structure and so we expect our results to be valid more generally. 


\section{B. Methods}

\section{Fixed Point Analysis}

Our goal is to understand the dynamical behaviour of the DALEC models. Previous studies have focussed on parameter fitting or measuring carbon fluxes by running the models forward in time.

Since we have made the drivers periodic on an annual cycle, this results in solutions of the DALEC model evolving towards periodic solutions with period of one year. These periodic states can be found either by running the model forward in time or by recognising that, for a periodic solution, the amount of carbon on day $t$ in year $n$ is the same as on day $t$ in year $n+1$ so, for example for the foliar carbon pool, a periodic solution will satisfy

$$
C_{f}(t)=C_{f}(t+365)
$$

Periodic solutions can therefore be found as fixed points of an annual map, which is obtained by iterating the daily map 365 times. We analyse the fixed points of the annual map and use continuation software to follow paths of fixed points. The advantages of finding the periodic states by solving for fixed points of the annual map are that it is then possible to trace out the behaviour of the periodic state as a function of the parameters, by varying either one or more parameters, and that it is possible to follow both stable and unstable solutions. We also determine the stability of the fixed points. Although unstable solutions are not seen in practice, they play a significant role as boundaries between regions in which qualitatively different behaviour occurs. From these results, the structure of the dynamics of the models can be determined.

The continuation software CL_Matcont_for_maps ${ }^{25}$, a freely available Matlab tool, is used to follow paths of fixed points of the annual map.

We now consider the stability of fixed points of the annual map for DALEC EV. The Jacobian matrix derived from the iteration (1)-(5) is given by

$$
J\left(C_{f}(t), C_{r}(t), C_{w}(t), C_{l i t}(t), C_{\text {som }}(t)\right)=
$$




$$
\left(\begin{array}{ccccc}
1-p_{5}+p_{3}\left(1-p_{2}\right) d G P P\left(C_{f}(t), t\right) & 0 & 0 & 0 & 0 \\
\tilde{p}_{4}\left(1-p_{2}\right) d G P P\left(C_{f}(t), t\right) & 1-p_{7} & 0 & 0 & 0 \\
\left(1-p_{3}-\tilde{p}_{4}\right)\left(1-p_{2}\right) d G P P\left(C_{f}(t), t\right) & 0 & 1-p_{6} & 0 & 0 \\
p_{5} & p_{7} & 0 & 1-\left(p_{8}+p_{1}\right) T(t) & 0 \\
0 & 0 & p_{6} & p_{1} T(t) & 1-p_{9} T(t)
\end{array}\right)
$$

where

$$
d G P P\left(C_{f}(t), t\right)=\frac{\mathrm{d} G P P\left(C_{f}(t), t\right)}{\mathrm{d} C_{f}(t)}
$$

We note that this matrix is lower triangular and that $1-\left(p_{8}+p_{1}\right) T(t)>0,1-p_{9} T(t)>0$ for all $t$ since the loss of litter or soil and organic matter cannot exceed the amount that is present.

The stability of a fixed point of the annual map is determined by the product of 365 such matrices, given by

$$
J^{a}\left(C_{f}(0), C_{r}(0), C_{w}(0), C_{\text {lit }}(0), C_{\text {som }}(0)\right)=\prod_{t=0}^{364} J\left(C_{f}(t), C_{r}(t), C_{w}(t), C_{\text {lit }}(t), C_{\text {som }}(t)\right)
$$

Now the product of lower triangular matrices is also lower triangular and so the matrix $J^{a}$ is lower triangular. Also, the diagonal entries of $J^{a}$ are the product of the diagonal entries of $J$. The stability of a fixed point of the annual map is determined by the eigenvalues of $J^{a}$ evaluated at the fixed point and since this matrix is lower triangular, its eigenvalues are the diagonal entries, which are given by

$$
\begin{gathered}
\lambda_{1}=\prod_{t=0}^{364}\left(1-p_{5}+p_{3}\left(1-p_{2}\right) d G P P\left(C_{f}(t), t\right)\right), \quad \lambda_{2}=\left(1-p_{7}\right)^{365}, \quad \lambda_{3}=\left(1-p_{6}\right)^{365}, \\
\lambda_{4}=\prod_{t=0}^{364}\left(1-\left(p_{8}+p_{1}\right) T(t)\right), \quad \lambda_{5}=\prod_{t=0}^{364}\left(1-p_{9} T(t)\right)
\end{gathered}
$$

We note that $0<\lambda_{2}, \lambda_{3}, \lambda_{4}, \lambda_{5}<1$ and that these eigenvalues do not depend on the annual fixed point. Thus, an annual fixed point will be stable if $\left|\lambda_{1}\right|<1$ or unstable if $\left|\lambda_{1}\right|>1$. Moreover, any bifurcations on a branch of fixed points are determined by $\lambda_{1}$ which depends only on the variable $C_{f}$. In Section III A, we consider the dynamics of the variable $C_{f}$ since this equation decouples from the remaining equations.

For the DALEC DE model, it can be shown that the Jacobian evaluated at an annual fixed point has the same four eigenvalues $\lambda_{2}-\lambda_{5}$ as for the evergreen model that lie between zero and one. Thus, the stability of a fixed point and any bifurcations on a branch of annual 

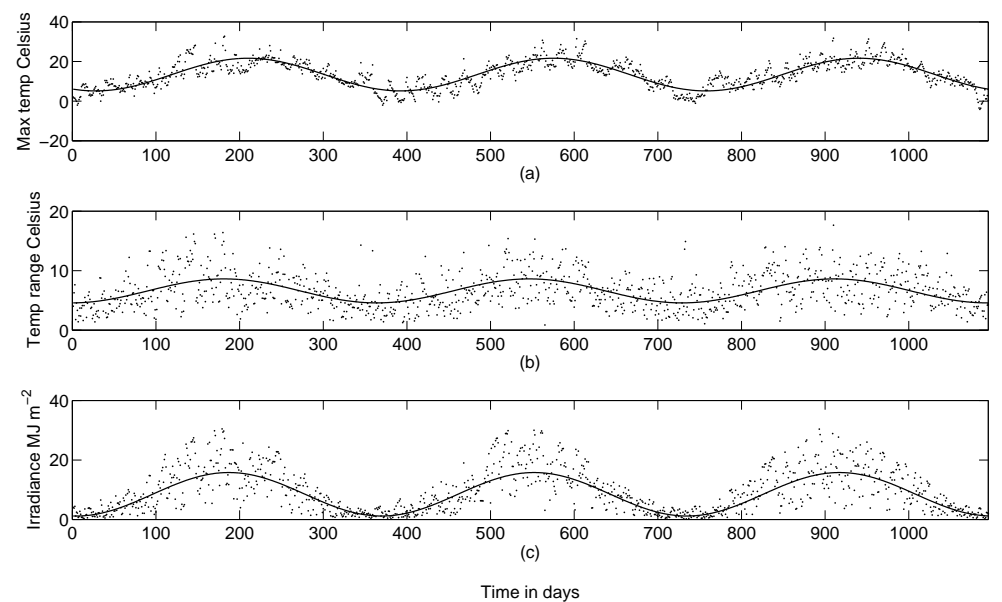

FIG. 3. Smooth and variable drivers over a three year period, data from Loobos forest in the Netherlands: (a) the maximum temperature, (b) the temperature range and (c) the irradiance. Note that the maximum temperature rarely drops below zero.

fixed points will be determined by the remaining $2 \times 2$ matrix derived from the $C_{f}$ and $C_{l a b}$ equations.

\section{Smoothing the Drivers}

The model is driven by highly variable drivers which are shown in Fig. 3. In Section III, we consider the effect that smoothing the variable drivers for temperature and irradiance has on the results. For example, we express the maximum temperature as:

$$
T_{\max }(t)=c_{0}+c_{1} \cos \left(\frac{2 \pi t}{365}\right)+c_{2} \sin \left(\frac{2 \pi t}{365}\right),
$$

which involves only three constants. For the temperature drivers a fast Fourier transform (FFT) of the data is used to find the constants $c_{0}, c_{1}$ and $c_{2}$. We found that the behaviour of $C_{f}$ was very sensitive to the irradiance and that smoothing the highly variable irradiance data with the simple periodic function given above using an FFT did not give a good prediction for $C_{f}$. Therefore, we used four dimensional variational (4DVAR) data assimilation to find values of $c_{0}, c_{1}$ and $c_{2}$ in the smooth approximation to the irradiance that gave good agreement with $C_{f}$ obtained using the driver data. We do not discuss the 4DVAR technique here, but it is described in many sources, such as Talagrand and Courtier ${ }^{26}$.

As well as describing the irradiance by three terms of a Fourier Series, we also describe 


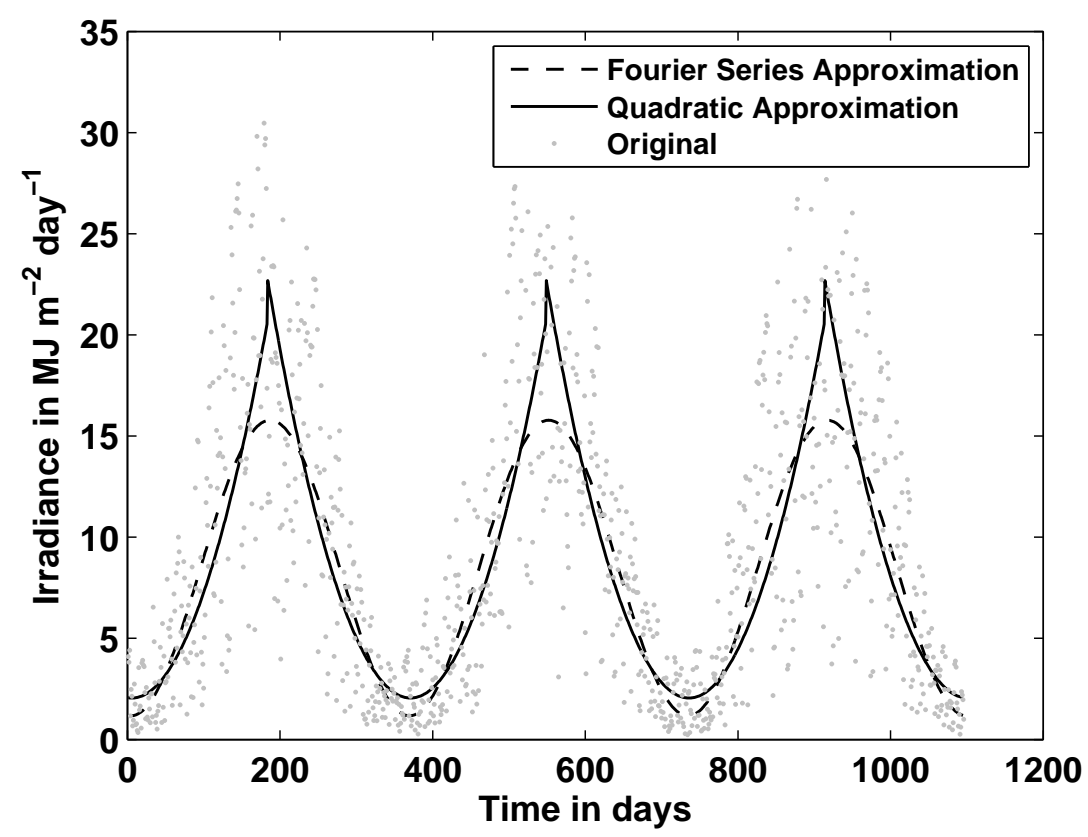

FIG. 4. The irradiance smoothed by a Fourier series and a quadratic function.

it by using a quadratic polynomial, created for one year and then periodically extended (by mapping it forward twice). The reason for this is that the Fourier series does not reach the higher peaks of the irradiance data (see Fig. 4).

\section{BEHAVIOUR OF THE CARBON POOLS FOR DALEC EV}

\section{A. Behaviour of the Foliar Carbon Pool}

We commence our analysis by observing that in the DALEC EV model equations (1)-(5), the equation for $C_{f}$ decouples from the other equations which means that the dynamics of the foliar carbon is independent of the other pools. In contrast, all the other pools are influenced by the behaviour of the foliar carbon pool. Therefore the dynamics of the forest is driven by and depends on $C_{f}$ and on the parameters in this pool, namely $p_{2}$ (fraction of GPP respired), $p_{3}$ (fraction of NPP allocated to foliage), $p_{5}$ (daily turnover fraction of foliage) and $p_{11}$ (nitrogen use efficiency parameter in the ACM). For the purpose of our analysis, we only consider parameters $p_{2}, p_{3}$ and $p_{5}$. Furthermore, in equation (1) for the foliar carbon pool the parameters $p_{2}$ and $p_{3}$ only appear in the combination $p_{3}\left(1-p_{2}\right)$; thus we define $p_{23}=p_{3}\left(1-p_{2}\right)$. Although the individual parameters $p_{3}$ and $p_{2}$ represent different 


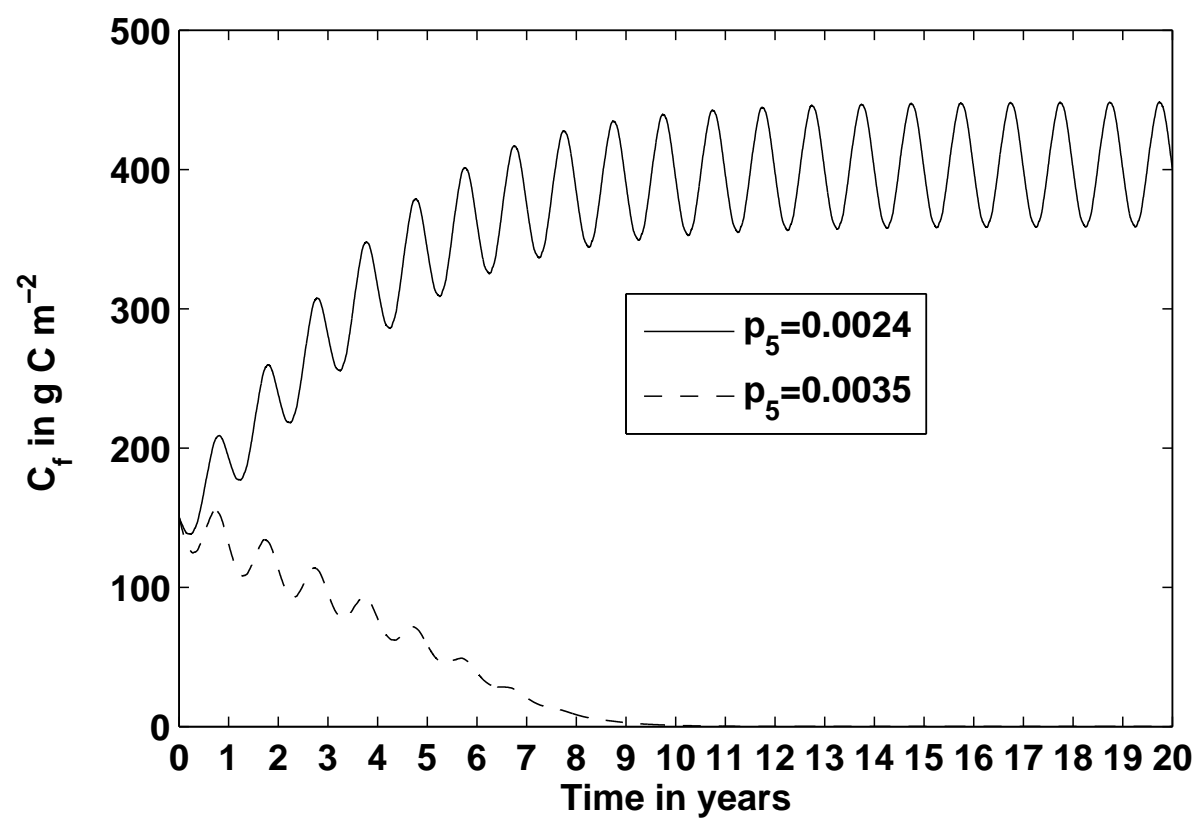

FIG. 5. The top line shows that for a value of $p_{5}=0.0024, C_{f}$ stabilises on a periodic state. The bottom line shows $C_{f}$ decreasing and going to zero for $p_{5}=0.0035$, showing several years of decline resulting in mortality. The values of the other parameters are $p_{23}=0.1392$ and $p_{11}=7.4$. Day 0 for each year is December $21^{\text {st }}$, the shortest day of the year at a latitude of $52^{\circ}$.

physiological processes, it is only their combination as $p_{23}$ that alters the dynamics of the foliar carbon pool. This parameter combination represents the fraction of GPP allocated to the foliar carbon every day.

Fig. 5 shows two graphs of $C_{f}$, resulting from running the model forward over a number of years using highly variable but periodic drivers, for two different values of $p_{5}$. The solid line $\left(p_{5}=0.0024\right)$ shows the foliar carbon growing to a stable periodic state with a period of one year. During this annual cycle there is more foliar carbon during the summer than during the winter, as photosynthesis is increased in the summer. In contrast, the dashed line $\left(p_{5}=0.0035\right)$ shows the foliar carbon steadily decreasing on average over a period of 8 to 9 years and then asymptoting to zero, which represents the forest dying. This raises the question: "How does the change in behaviour take place and at what parameter value?"

The transient behaviour of $C_{f}$ that leads to a stable periodic state, as shown in Fig. 5, suggests that the DALEC EV model has a periodic solution with a period of one year for certain values of the parameters. As described in Section II B, this periodic solution can be 


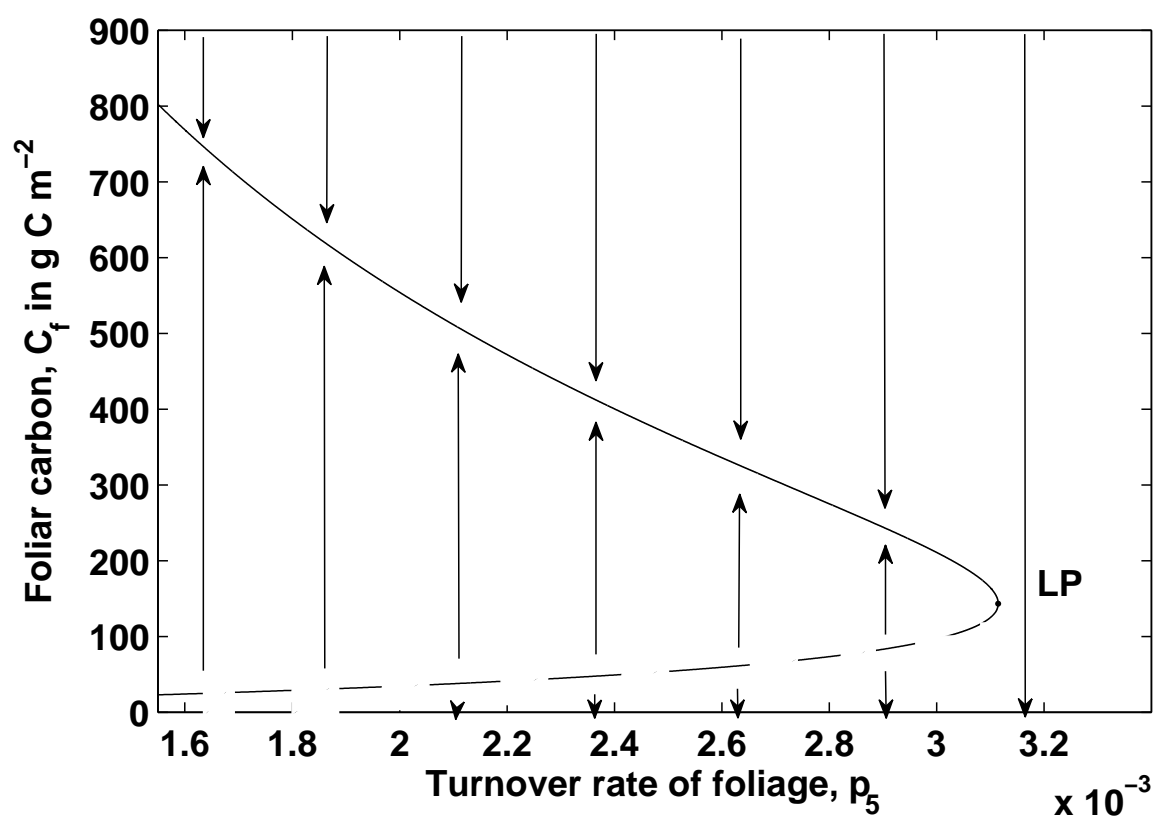

FIG. 6. Path of fixed points for the annual map for $p_{23}=0.1392$. The top branch is stable and the bottom branch is unstable and there is also a stable fixed point branch at $C_{f}=0$. On the vertical axis the value of $C_{f}$ on day 0 of each year (December $21^{\text {st }}$ ) is shown. The arrows indicate how solutions are drawn towards the stable and away from the unstable branches over time.

regarded as a fixed point of an annual map and so we used CL_Matcont_for_maps ${ }^{25}$ to find this fixed point and to follow a path of fixed points with varying $p_{5}$. The results in Fig. 6 show how the periodic state changes as parameter $p_{5}$ is varied. It has two branches, representing two paths of fixed points, which are connected by a limit point or fold bifurcation. The top branch is stable and the bottom branch is unstable, with the change of stability occurring at the limit point. If a fixed point is stable, it acts as an attractor and so solutions close by will be drawn towards this fixed point solution. If a fixed point is unstable, it acts as a repellor for nearby solutions.

We have already seen in Section II A that $\operatorname{GPP}(0, t)=0$ for all $t$ and this implies that $C_{f}(t)=0$ is a fixed point of the daily map and therefore is also a fixed point of the annual map for all values of the parameters. The derivative of the iteration for the annual map with respect to $C_{f}(t)$, evaluated at $C_{f}(t)=0$, is $\left(1-p_{5}\right)^{365}\left(\right.$ since $\left.G P P\left(C_{f}, t\right)=O\left(C_{f}^{2}\right)\right)$ and so, since $0<p_{5}<1$ (which is always true for physical reasons), the zero fixed point is always linearly stable. In this way, we find that the annual map of $C_{f}$ has either one (stable) 
fixed point or three fixed points (two stable separated by one unstable), depending on the parameter values.

Unstable solutions are not seen in simulations, but they play a significant role in determining what kind of behaviour occurs. For example, if a simulation is carried out with the initial value of $C_{f}(0)=C_{f_{0}}$ at a point anywhere on the graph between the top and bottom branch, such as at $p_{5}=0.0024$ and $C_{f_{0}}=140$ (see Fig. 5), then $C_{f}$ will be attracted to the top branch and will grow to a stable periodic state. If the initial condition is at a point below the bottom branch, for example at $p_{5}=0.0024$ and $C_{f_{0}}=20$, it will be repelled from the bottom branch and attracted to the fixed point $C_{f}=0$. If $p_{5}$ is chosen to the right of the limit point, for example $p_{5}=0.0035$ (see Fig. 5) then the iterates will also be attracted to $C_{f}=0$ for all initial values of $C_{f}$. In this case, the forest is unsustainable and will eventually die for any initial value of the foliar carbon (see Fig. 5). Once the forest has died, it is not possible for it to re-grow, according to the model, as the state $C_{f}=0$ is an attractor.

In Fig. 7 the limit point of $C_{f}$ is shown for a range of values of $p_{5}$ and $p_{23}$. If $p_{23}$ changes, the path of fixed points will move and the limit point will move with it. In this way one can imagine a whole series of new curves with new limit points. Fig. 7 shows the line of limit points along with a schematic series of curves. We used CL_Matcont_for_maps ${ }^{25}$ again, varying $p_{23}$ as well as parameter $p_{5}$, to compute a line of limit points. The computed surface of fixed points and the line of limit points is shown in Fig. 8.

By finding the limit points of all the possible parameter combinations it is possible to define for which values of the parameters a forest has a chance of growing to a periodic state and living, depending on the initial value of $C_{f}$, and for which values the forest is doomed to widespread mortality. As $C_{f}$ is central to the distribution of carbon to the different carbon pools, it is possible to conclude that for values of $p_{23}$ and $p_{5}$ situated on the left hand side of the line of limit points, the evergreen forest either grows to a stable periodic state and lives or it will eventually die, depending on the initial condition for $C_{f}$. For values of the parameters on the right hand side of the line of limit points the conditions are such that an evergreen forest will eventually die whatever the initial value of $C_{f}$. This answers the question on how the behaviour of the foliar carbon pool changes for different values of the parameters, $p_{2}, p_{3}$ and $p_{5}$. It is interesting to note that the parameter values we have used result in a point near the limit point line, see Fig. 7. Our choice of parameter values came from the REFLEX study. As part of this study nine different groups estimated parameters 


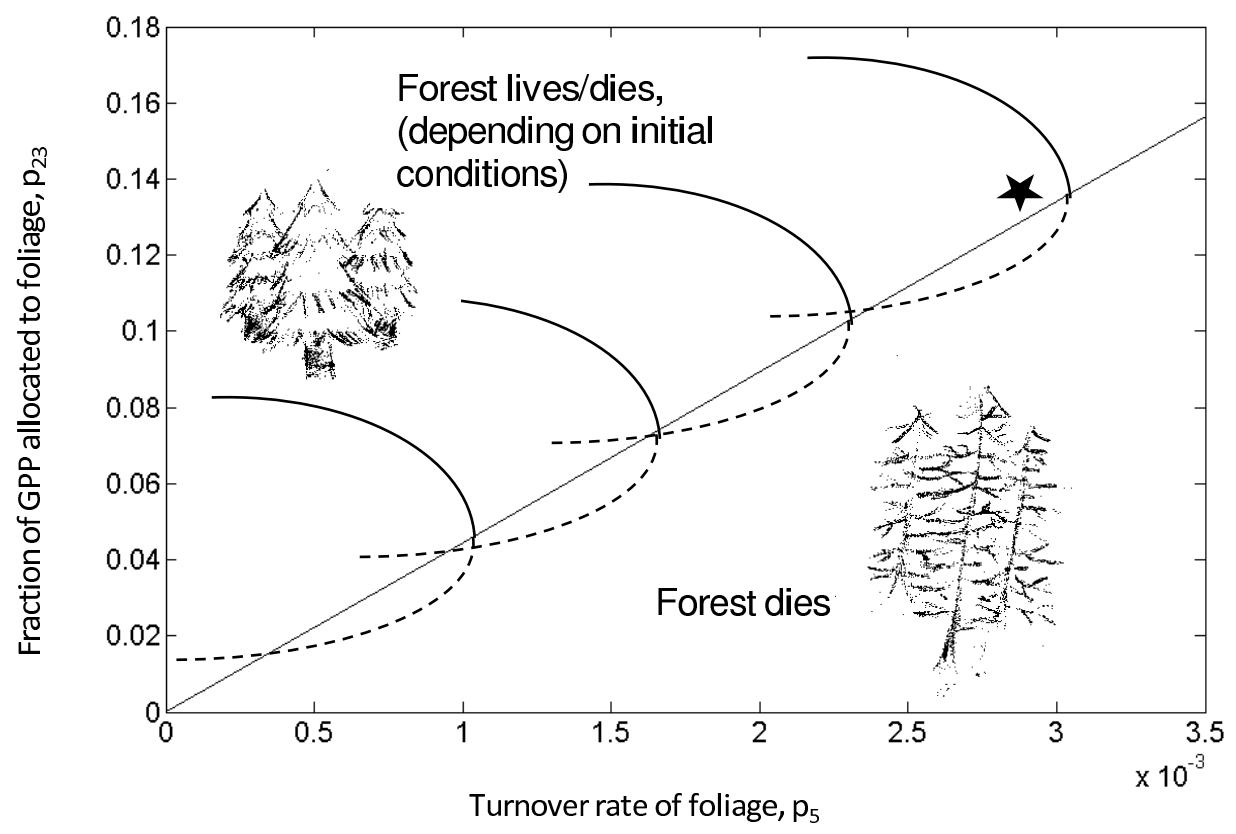

FIG. 7. Schematic diagram showing how the path of limit points divides the parameter plane into two different regions. Note the star near the line of limit points, which represents realistic parameter values: $p_{5}=0.0028$ and $p_{3}\left(1-p_{2}\right)=0.1392$.

for the DALEC evergreen model from synthetic data of LAI and NEE. From Table 5 in ${ }^{19}$, we see that estimates for the parameter combination $p_{23}$ varied from 0.08 to 0.19 and for $p_{5}$ from 0.0027 to 0.004 . The synthetic data for the study was generated by running the DALEC model forward in time for the parameters indicated by the star in Fig. 7, a region where the model predicts that the forest will grow to a steady oscillatory state: seven of the nine groups estimated parameters which indicated that the forest was dying.

We used climate and parameter data from another, younger, forest to see if the parameter values would also result in a point near the limit point line. The forest is a Metolius young ponderosa pine site located in a Research Natural Area in the Eastern Cascades, near Sisters, Oregon, USA ${ }^{21}$, which, similarly to the Loobos forest used in the REFLEX project, enjoys a temperate climate. Nearly all the parameter values for this forest are different, including the $a$-parameters in the ACM, see Appendix D. The star in Fig. 9 is again near the line of limit points; close enough that again uncertainty in the parameter values could result in different predictions as to the future fate of the forest. 


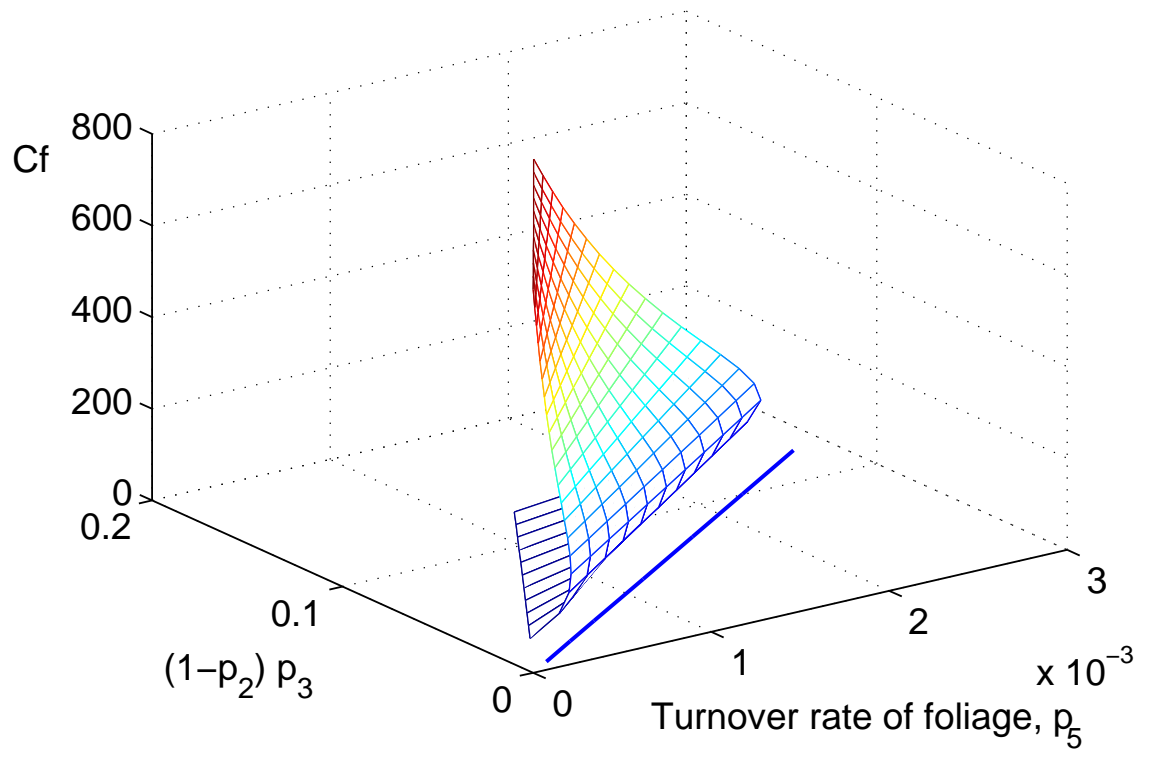

FIG. 8. A plot of the surface of fixed points with the line of limit points projected onto the parameter plane.

A possible reason for this observed behaviour is that if the mean lifetime of the leaves or needles $\left(p_{5}\right)$ is controlled by physiological factors, and hence comparatively inflexible, then the strategy adopted by a tree to give maximum growth would be to minimise the quantity of carbon allocated to leaves $\left(p_{23}\right)$ while remaining viable. This suggests that it should lie close to but above the line of limit points.

\section{B. Smoothing the Drivers}

The drivers for temperature and irradiance commonly used are highly variable, see Fig. 3. We now consider the effect that smoothing the drivers has on the results as, for mathematical analysis purposes, it is more desirable to work with simple functions rather than raw data. The smoothed drivers using the three term Fourier series approximation obtained using the methods described in Section II B 2 are shown in Fig. 3. The Fourier series approximation and the piecewise quadratic approximation to the irradiance are shown in Fig. 4. We found that although with the quadratic approximation the higher peaks are reached, there was not much difference between this approximation and the Fourier series approximation when 


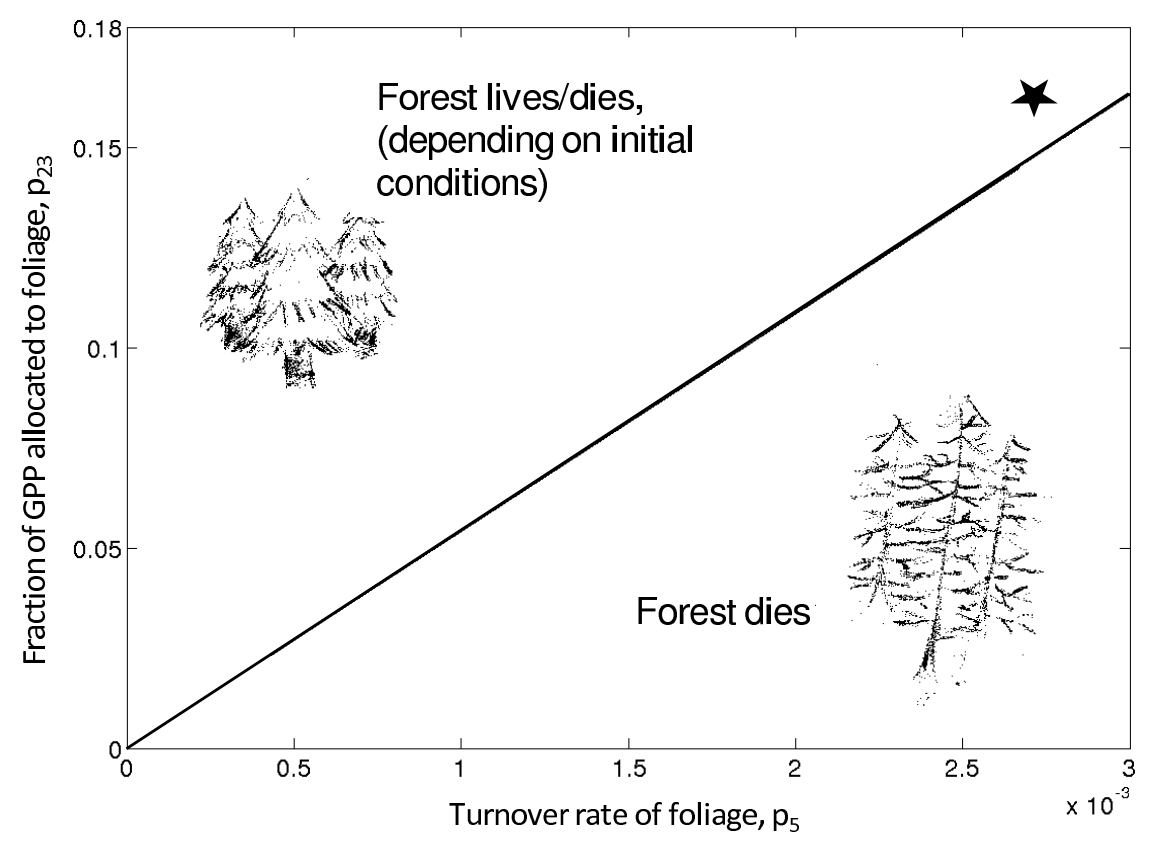

FIG. 9. The line of limit points for the Oregon forest. The star represents the parameter values. For this forest $p_{5}=0.0027$ and $p_{3}\left(1-p_{2}\right)=0.1659$.

compared, running forward the $C_{f}$ equation. Therefore, for the sake of simplicity, we have used the Fourier series approximation in our analysis.

In Fig. 10 the graph for $C_{f}$ is obtained by running the model forward over three years, both with the original highly variable drivers and the smoothed drivers. This shows that the the effect of the smoothing on $C_{f}$ is minimal. The path of fixed points and the line of limit points produced using the smoothed drivers also show qualitatively very similar behaviour, see Figs 11 and 12. The only difference is a small change in the path of fixed points for the foliar carbon and in the position of the limit point. The use of smoothed drivers greatly simplifies the model as expressing data in terms of functions means it is not necessary to use separate data files. It can also simplify data collection as only three parameters are required, namely the mean, amplitude and phase of the annual cycle. The fact that smoothing does not qualitatively change the dynamics illustrates the robustness of the limit point structure.

According to ${ }^{27}$, natural variability in drivers is important and ecosystems respond to it. They show that by taking away high-frequency variability decadal Net Ecosystem Production (NEP), GPP and total respiration are significantly enhanced. However, the ACM only takes into account daily measures of climate data and therefore the high frequency variability has 


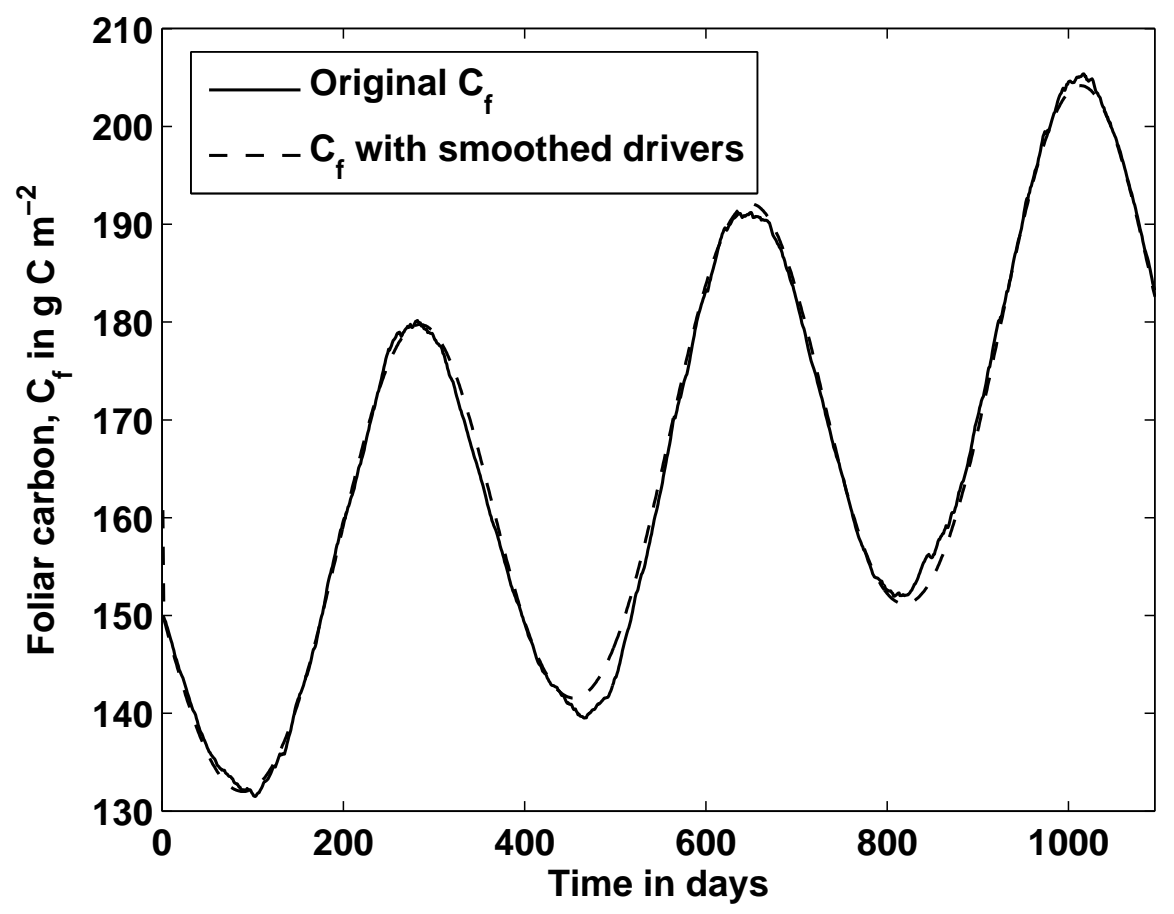

FIG. 10. $C_{f}$ over three years shown using noisy drivers and smoothed drivers.

already been taken out. This could also explain why, in Figs 11 and 12, we do not see the effect predicted $b y^{27}$.

\section{Variation in the Atmospheric Carbon and Temperature}

In our model we have assumed that the drivers are periodic on an annual timescale and that the atmospheric carbon is constant. However, in reality the atmospheric carbon is increasing year by year partly due to human activities such as the burning of fossil fuel ${ }^{28}$. The effect of atmospheric carbon is included in the model of photosynthesis, see equation (B2) in Appendix B. Fig. 13 shows the effect that raising the atmospheric carbon, $C_{a}$, has on the curve in Fig. 11 (using the smoothed drivers). For a given value of $p_{5}$, if there is more $\mathrm{CO}_{2}$ available for photosynthesis, then the forest grows towards a higher periodic solution. The region in the parameter plane where a forest can live is also bigger as the limit point moves to the right as $C_{a}$ increases.

Interestingly, the increase of mean daily temperature by a constant amount of $2.4^{\circ} \mathrm{C}$ has no significant effect on the line of limit points. Several studies suggest that this is the 


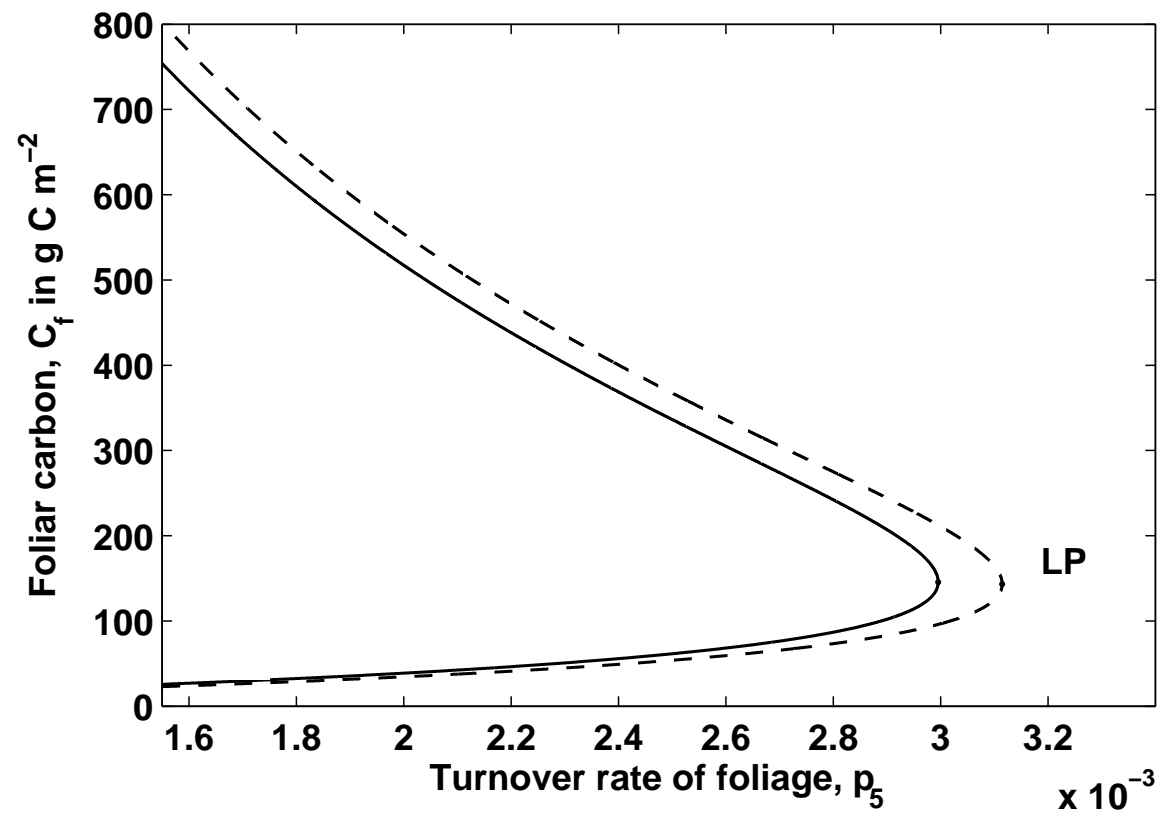

FIG. 11. The solid curve is the result of using smoothed drivers, the dashed curve is the result of using noisy drivers.

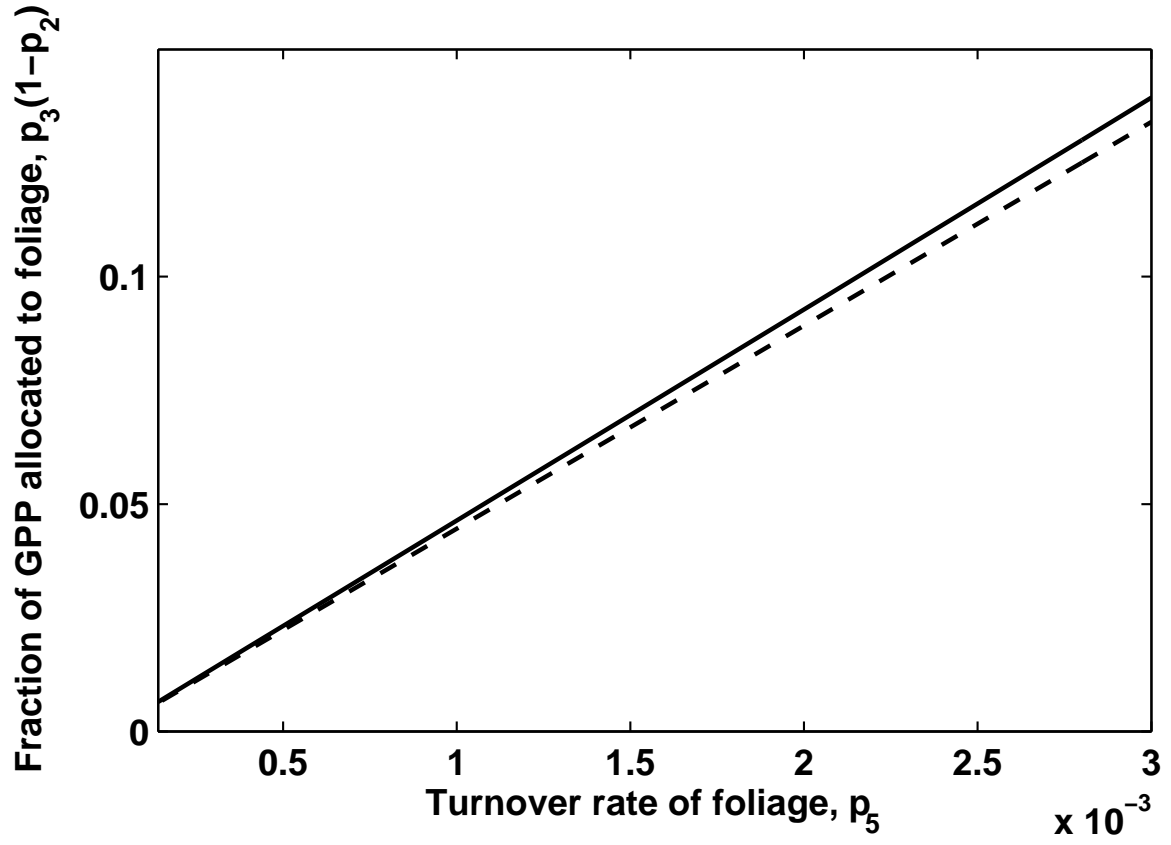

FIG. 12. The solid line of limit points is the result of using smoothed drivers, the dashed line is the result of using noisy drivers. 


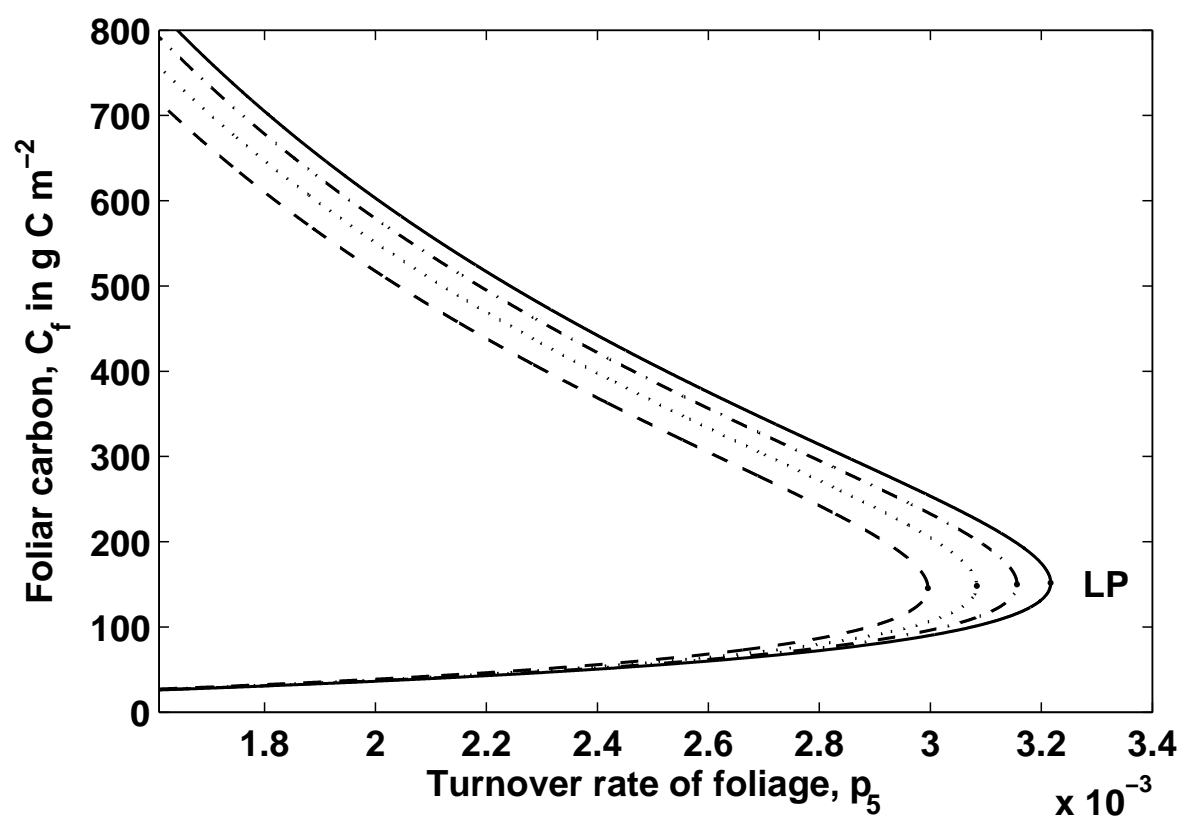

FIG. 13. Paths of fixed points for $C_{a}=380$ (dashed), $C_{a}=430$ (dot), $C_{a}=480$ (dash-dot) and $C_{a}=530$ (solid).

approximate amount the temperature will rise by the end of the $21^{\text {st }}$ century even if the atmospheric carbon level is kept at its current value ${ }^{29}$. This lack of effect could be due to the fact that the data used is from a forest in a moderate climate. There are only 23 days in the data where the temperature during the day is slightly below zero. In DALEC EV no provision is made for when the temperature goes below zero, which is when photosynthesis slows down or stops ${ }^{30}$. In DALEC EV photosynthesis takes place whatever the temperature. One might anticipate more temperature dependence for deciduous forests, as a temperature increase here could result in an increase in the length of the growing season.

\section{Behaviour of the Other Carbon Pools}

For each of the fixed points of the annual map for the foliar carbon pool we can find corresponding fixed points for the other four carbon pools. For the fixed point $C_{f}=0$ it can be shown that all other pools also have a fixed point at zero. The Jacobian matrix of equations (1)-(5) evaluated at this fixed point is lower triangular with diagonal entries that are all less than one, and so the fixed point in which all the pools are zero is a stable fixed point of the model. 


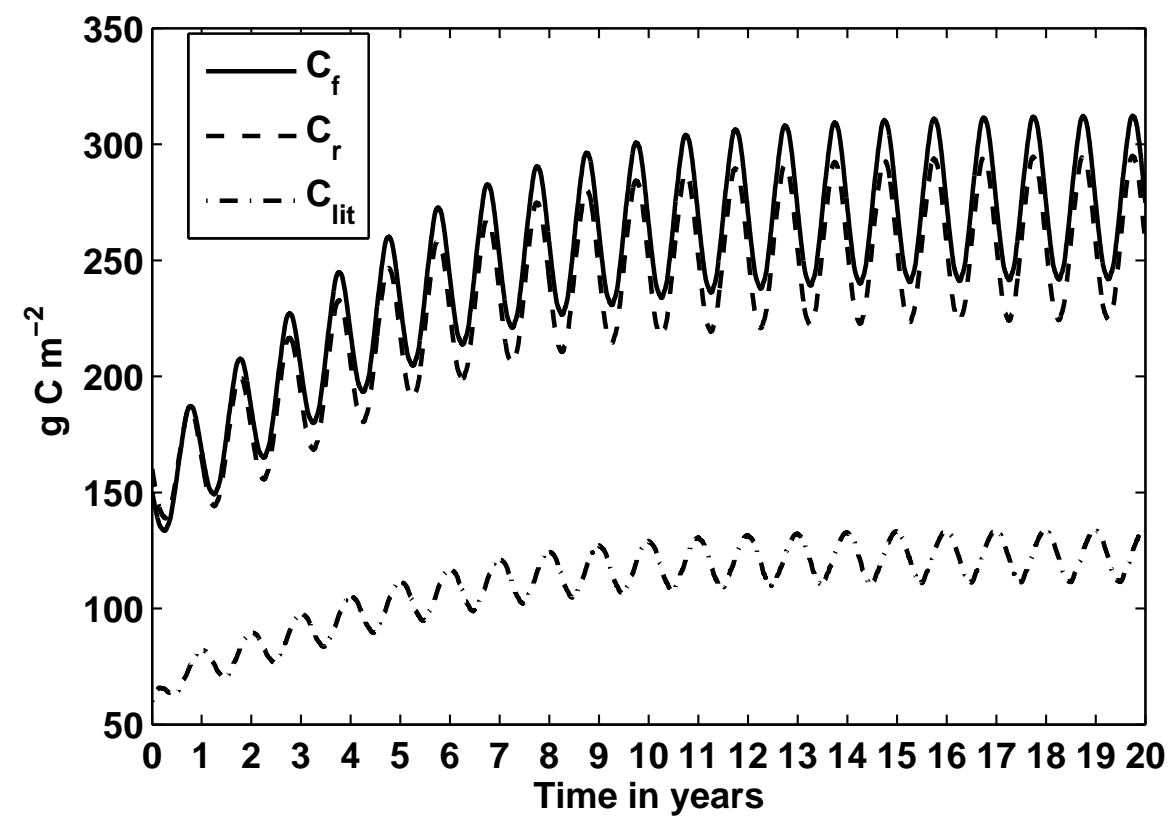

FIG. 14. Behaviour of $C_{f}, C_{r}$ and $C_{\text {lit }}$ over 20 years; all three of these carbon pools reach a periodic state within this time.

The roots carbon pool, $C_{r}$, and the litter carbon pool, $C_{l i t}$, reach their (non-zero) fixed points relatively quickly, on the same time scale as the foliar carbon pool, as the parameters $p_{7}$ and $p_{8}$ are not too small, see Fig. 14. However, the soil and organic matter carbon pool, $C_{\text {som }}$, and the woody carbon pool, $C_{w}$, take a very long time (thousands of years) to reach their fixed points, due to the very small values of the parameters $p_{6}$ and $p_{9}$, see Fig. 15 . The wood pool, $C_{w}$, grows faster than the soil and organic matter pool. Due to the very slow growth of $C_{\text {som }}$, we can regard the initial value of $C_{\text {som }}$ as a pseudo-fixed point on a decadal scale.

\section{PRELIMINARY RESULTS FOR DALEC DECIDUOUS}

The main difference between the evergreen and deciduous models is that DALEC DE has an extra labile pool, $C_{l a b}$, which is used in the spring to kickstart the growth of leaves. It depletes as the leaves grow and then builds up again in the autumn, when the leaves are dropping. In this case, we see from the model equations (6)-(11) that the foliar and labile pools are coupled but decouple from the other four equations.

For the parameter values given in Appendix A we found that $C_{f}$ and $C_{l a b}$ go to zero, 


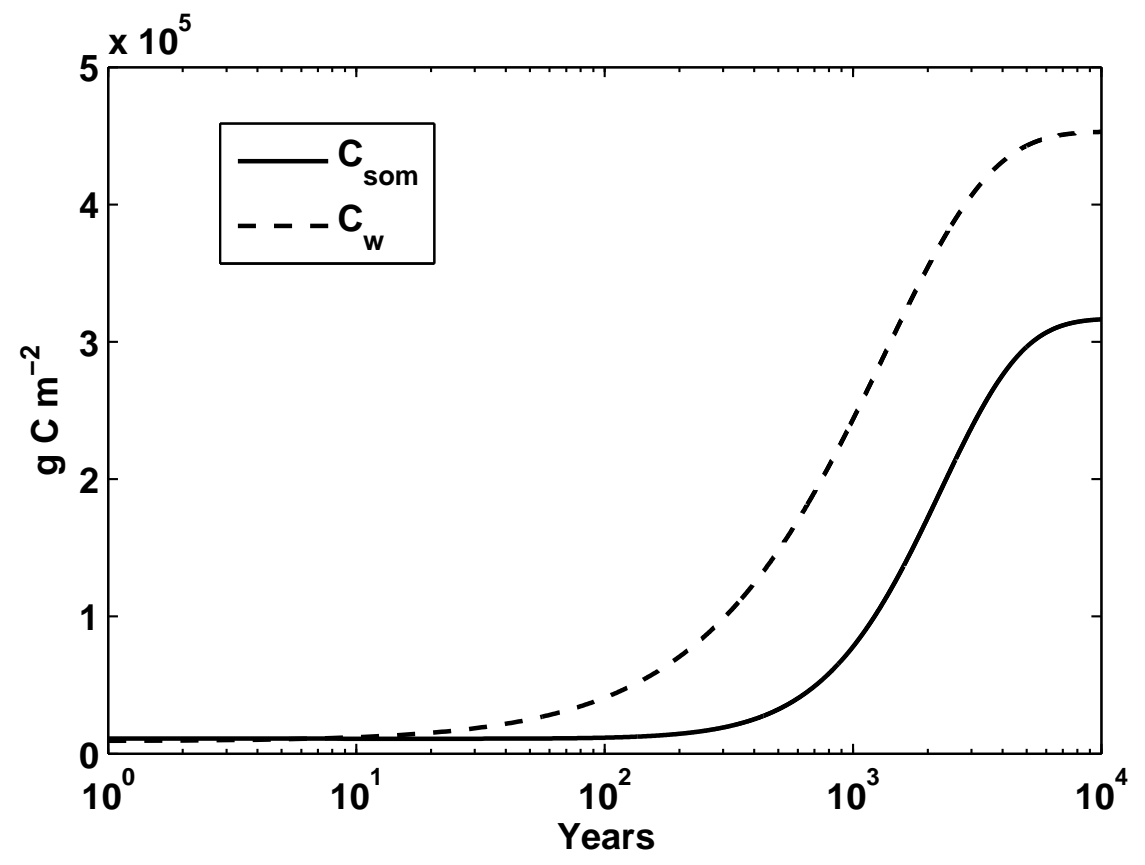

FIG. 15. Only one point per year plotted, removing the periodic behaviour: $C_{\text {som }}$ and $C_{w}$ do not reach their fixed points until after 10,000 years (note the log scale).

which corresponds to the forest dying. Thus, we changed the parameter $p_{14}$ to be $p_{14}=0.19$ and found that $C_{f}$ and $C_{l a b}$ then converge to a positive periodic cycle, which corresponds to the forest living. Thus, it seems that there is a similar structure in this model to that of DALEC EV. Fig. 16 shows the foliar and labile pools plotted over one year with $p_{14}=0.19$. In the spring, approximately between days 100 and 150, foliar turnover is switched off and labile turnover is switched on, allowing leaves to start growing again. This depends on temperature and the time of year. In the summer, both the labile pool and the foliar pool remain unchanged for some time (approximately between days 200 and 250), which is when both the foliar turnover and the labile turnover are switched off. In the autumn, foliar turnover is switched on, when the minimum temperature goes below the value of $p_{13}$ and after more than 200 days of the year have passed. During this phase, the trees drop their leaves and photosynthesis comes to a halt and so the foliar carbon pool is depleted and carbon is stored in the labile pool until spring.

Even though the dynamics of the forest now depends fundamentally on the two carbon pools $C_{f}$ and $C_{l a b}$, we find a bifurcation structure which is similar to that of DALEC EV, see Fig. 17. We used the variable daily climate data for these results. However, this time, as 
it is important that the foliar carbon depletes to zero in the autumn and the labile carbon depletes to zero in the spring, we use parameter $p_{14}$, the fraction of carbon loss to litter, as our bifurcation parameter. Varying this parameter retains the depletion of both pools whereas increasing $p_{5}$ can stop the foliar carbon pool going to zero in the autumn. So when we keep all the other parameters fixed and vary $p_{14}$, we find a limit point for the coupled dynamics of the foliar carbon pool $C_{f}$ and the labile carbon pool, $C_{l a b}$, see Figs 17 and 18 . It is interesting to note that the top branches of both graphs are much steeper than the top branch in Fig. 6. A small change in $p_{14}$ causes a large change in the value of the fixed point. However, we also note that $C_{f}$ is very small, since we are sampling during the winter on $21^{\text {st }}$ December each year.

We also see from equations (6) and (7) that $C_{f}=C_{l a b}=0$ is a fixed point of the daily map and hence also of the annual map and so, as with DALEC EV, there are either one or three fixed points depending on the parameter values. For DALEC EV we showed that the trivial fixed point $C_{f}=0$ is linearly stable. We now show that for DALEC DE the fixed point $C_{f}=C_{l a b}=0$ is also linearly stable.

We write the iteration given by (6) and (7) as

$$
X_{n+1}=F\left(X_{n}, t\right)
$$

where $X=\left(C_{f}, C_{l a b}\right)$ and $F(0, t)=0$. If the Jacobian matrix for these equations is $J(X, t)$, then the trivial fixed point will be linearly stable if $\rho(M)<1$, where $\rho$ is the spectral radius and

$$
M=\prod_{t=0}^{364} J(0, t)
$$

The form of $J(0, t)$ depends on the values of the two switches $m_{t f}$ and $m_{t l}$. In spring, $m_{t f}=0$ and $m_{t l}=1$ and then

$$
J(0, t)=J_{s}(0, t)=\left[\begin{array}{cc}
1 & p_{15}\left(1-p_{16}\right) T(t) \\
0 & 1-p_{15} T(t)
\end{array}\right]
$$

In summer, $m_{t f}=m_{t l}=0$ and then $J(0, t)=I$. Finally, in autumn and winter, $m_{t f}=1$ and $m_{t l}=0$ and then

$$
J(0, t)=J_{w}(0, t)=\left[\begin{array}{cc}
1-p_{5}\left(p_{14}+\left(1-p_{14}\right) T(t)\right) & 0 \\
p_{5}\left(1-p_{14}\right)\left(1-p_{16}\right) T(t) & 1
\end{array}\right]
$$

We note that the model requires all the non-zero entries in these matrices to be positive. Taking the matrix one-norm of these matrices, we then see that $\|J(0, t)\|_{1}=1$ in all cases. Thus, using standard properties of matrix norms, we have 


$$
\rho(M)=\rho\left(\prod_{t=0}^{364} J(0, t)\right) \leq\left\|\prod_{t=0}^{364} J(0, t)\right\|_{1} \leq \prod_{t=0}^{364}\|J(0, t)\|_{1}=1
$$

This does not quite give us the result that we want, as we require strict inequality, and so we need to introduce at least one strict inequality. To do this, we note that winter will turn to spring and so, for some $t$, there will be a matrix product $J_{s}(0, t+1) J_{w}(0, t)$. We aim to show that $\left\|J_{s}(0, t+1) J_{w}(0, t)\right\|_{1}<1$ as this is then sufficient to give $\rho(M)<1$ as required.

To verify this inequality, we first note that since all the entries in both matrices are nonnegative, as noted above, then all the entries in the matrix product $J_{s}(0, t+1) J_{w}(0, t)$ will also be non-negative. Thus the one-norm of this product is simply the largest column sum with no need to take absolute values. The sum of the second column is simply $1-p_{15} p_{16} T(t)$ while the sum of the first column is given by $1-p_{5} p_{14}-p_{5} p_{16} T(t+1)\left(1-p_{14}\right)\left(1+p_{15}\left(1-p_{16}\right) T(t)\right)$. These are both strictly less than one and so $\left\|J_{s}(0, t+1) J_{w}(0, t)\right\|_{1}<1$ as required.

Thus, we conclude that the trivial fixed point $C_{f}=C_{l a b}=0$ is linearly stable as claimed. It is then follows from the analysis in Section II B 1 that the fixed point for the full model where all the pools are zero is a stable fixed point.

\section{DISCUSSION AND CONCLUSION}

We have analysed the qualitative dynamical behaviour of DALEC EV and DALEC DE, which are simple process-based vegetation models for carbon stocks and stores of evergreen and deciduous forests that have been used previously to study model uncertainty, parameter estimation and carbon fluxes. We have shown that the dynamics of the DALEC EV model fundamentally depends on the behaviour of the foliar carbon pool and the parameters involved in this pool, $p_{3}\left(1-p_{2}\right)$ (fraction of GPP allocated to foliage), $p_{5}$ (daily turnover rate of foliage) and $p_{11}$ (nitrogen use efficiency parameter in the ACM). The dynamics of the foliar carbon pool have a limit point that is dependent on the value of these parameters. For appropriate values of the parameters and initial conditions the carbon pools converge to stable periodic annual cycles. However, if either the allocation of carbon to foliage is too small or the foliage turnover rate is too large then the model predicts that widespread mortality occurs. Our preliminary study of DALEC DE found that it exhibits a similar limit point structure to the evergreen model.

We note that if we had not removed the constraint that LAI is $\max \left(0.1, C_{f} / \operatorname{lma}\right)$ and 


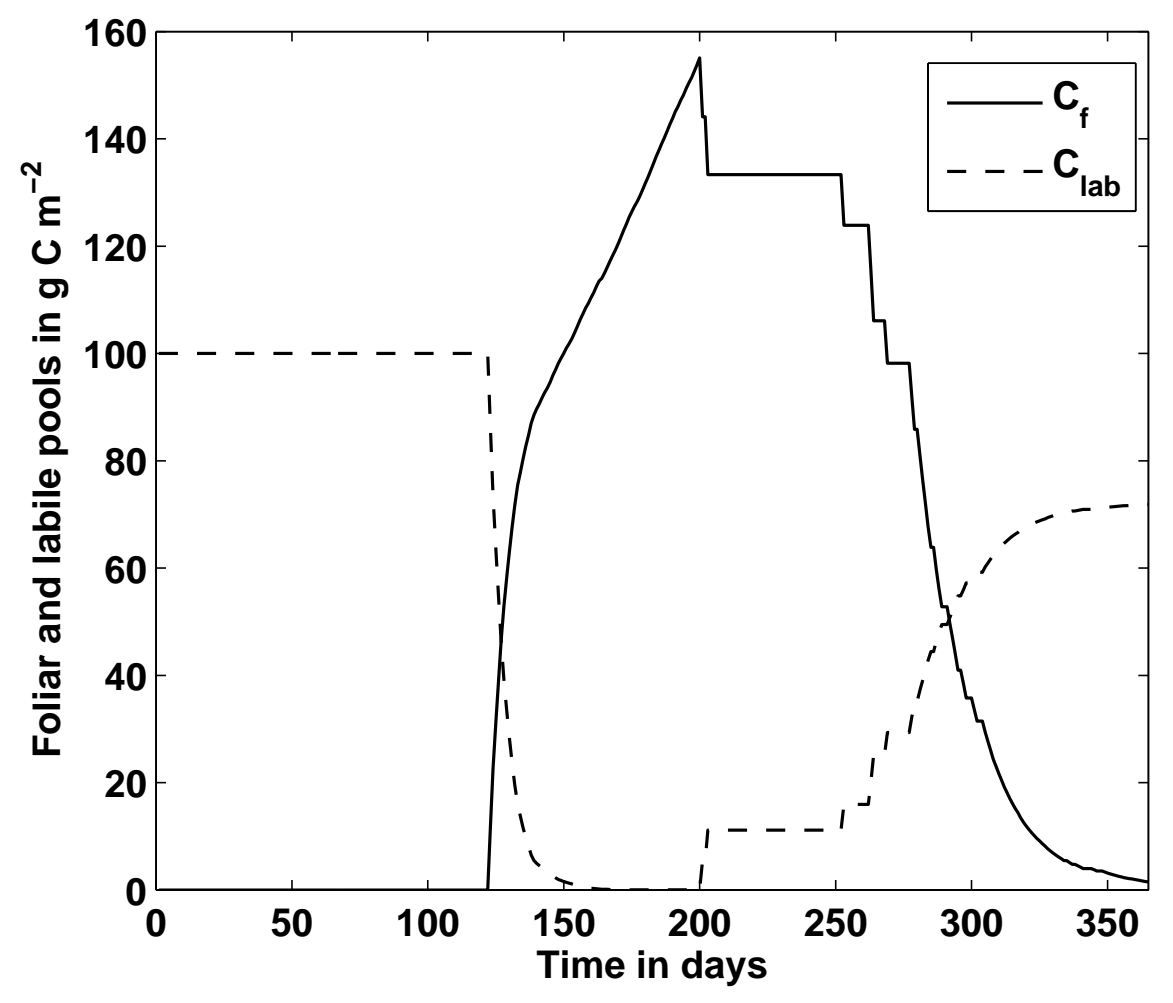

FIG. 16. The foliar and labile pools plotted over one year with $p_{14}=0.19$.

replaced it with $\mathrm{LAI}=C_{f} /$ lma in the DALEC evergreen model, then the limit point structure would be unchanged but $C_{f}=0.1 \times$ lma would play the same role as $C_{f}=0$ does in our work.

That the allocation of carbon to foliage has to be in balance with the foliage turnover rate for a forest to survive is perhaps not surprising. But the fact that typical parameter values for the two evergreen forests that we have considered, the Loobos site in the Netherlands and the NRA site near Sisters, in Oregon, USA, sit near a limit point is interesting. One consequence is that if for any reason, such as sustained drought, parameters in the model change then forests can 'tip' from a stable to an unstable state resulting in widespread mortality. In Section IIIA a proposed reason for why evolutionary pressures might drive typical parameter values towards the limit point was given. The fact that typical parameter values are above and not at the limit point is a consequence of the fact that long term survival requires that typical climatic conditions do not cause such tipping.

The presence of the limit point has other consequences: given a level of uncertainty in the parameters that is not untypical, it is difficult to predict even whether the forests that 


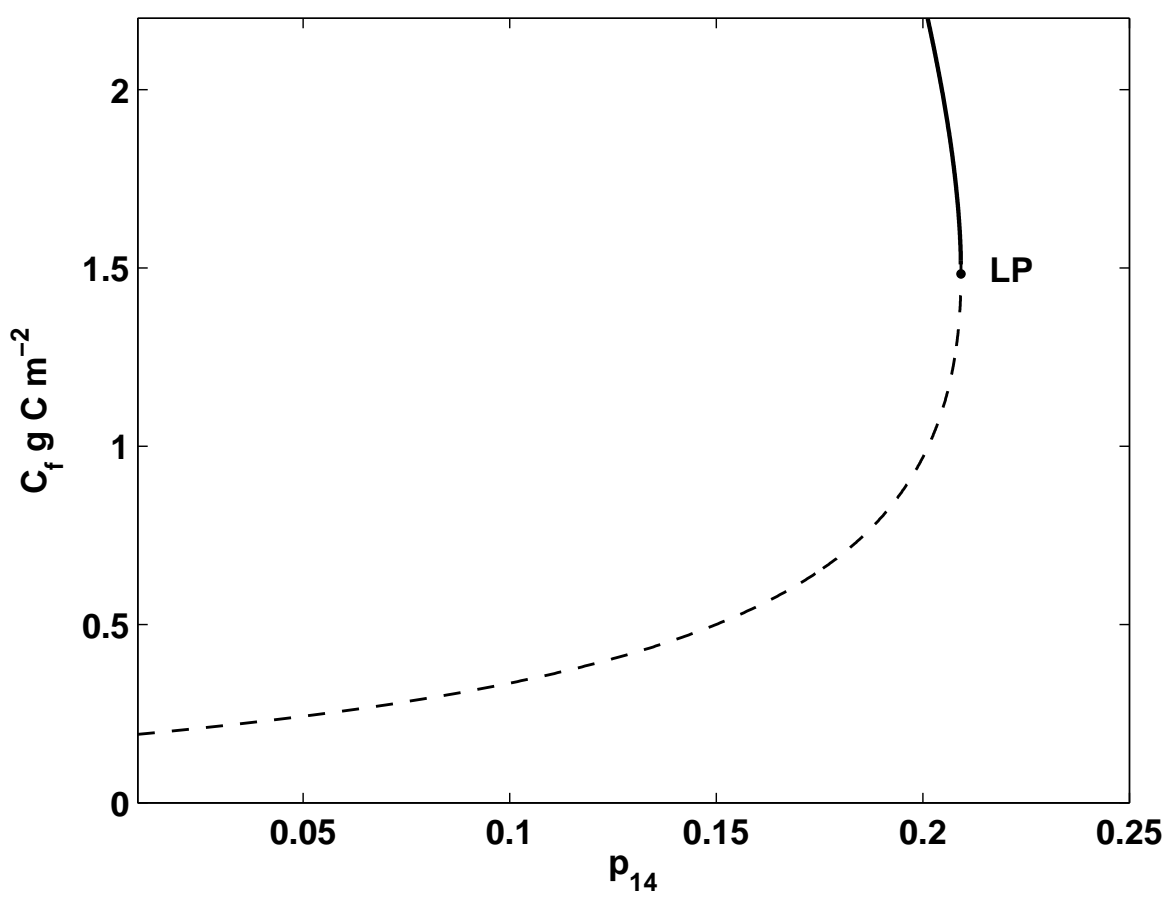

FIG. 17. Limit point for parameter $p_{14}$ on a path of fixed points of the annual map for $C_{f}$ for DALEC DE. The top branch is stable, the bottom branch is unstable and there is also a stable fixed point branch at $C_{f}=0$. The fixed points are sampled on day 0 of each year, which is December $21^{\mathrm{st}}$.

are being modelled are growing or dying. This is illustrated by the fact that the parameters predicted by REFLEX project participants using exactly the same data, in some cases predicted a growth in foliar carbon and sometimes a decline. This occurs for both evergreen and deciduous versions - in the latter case this is visible in Fig. 7 of $^{19}$ where, although most groups predict that the foliar carbon is decreasing, at least one group predicts that it is increasing. This is not a fault of the simplicity of the model, but a result of the fact that there is a structural change in the underlying dynamical system. Knowing where such structural changes occur highlights parameter regimes where even getting the trend correct is challenging. Such structural changes may also have implications for the practical implementation of data assimilation schemes since, close to limit points, the model state variables become very sensitive to the bifurcation parameters.

The fact that in the DALEC evergreen model the foliar carbon pool decouples from the other pools has important consequences for data assimilation using this model. A direct 


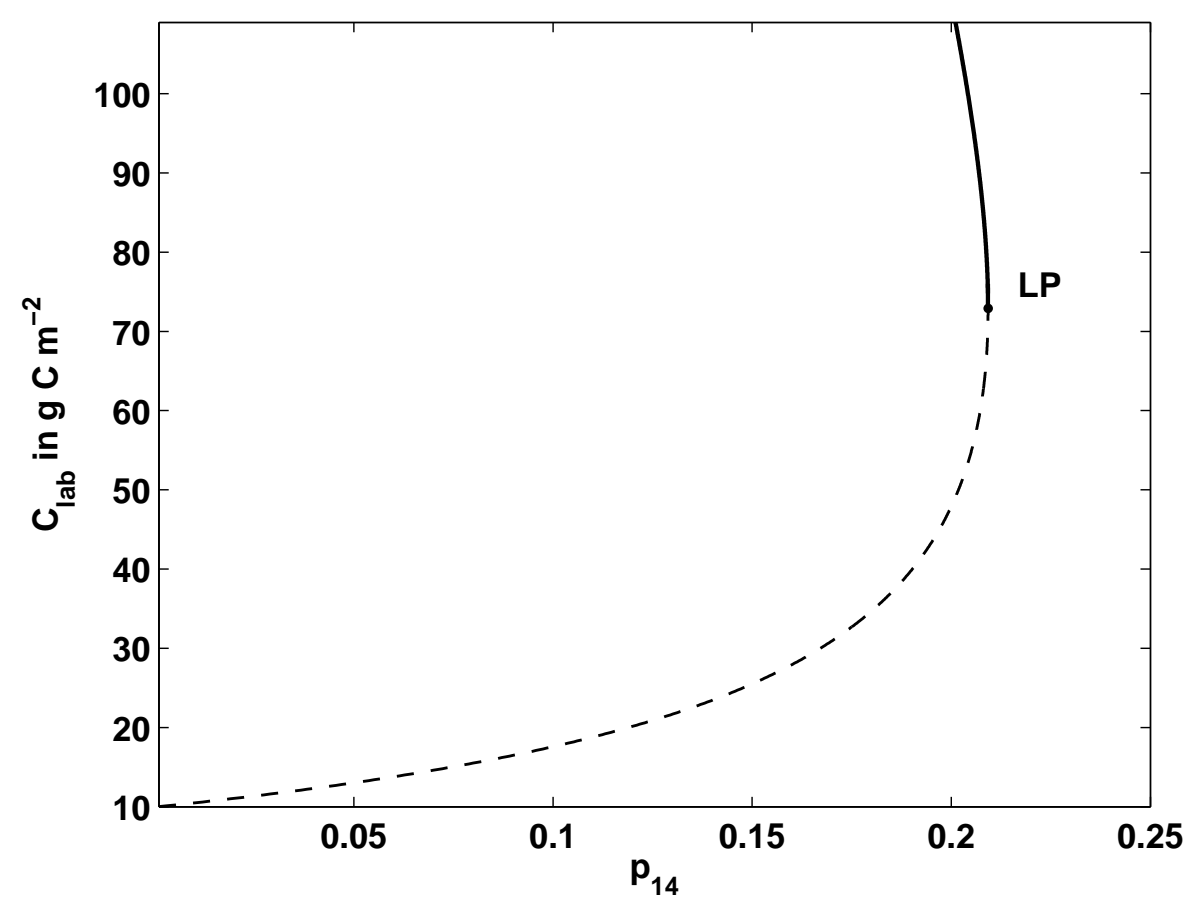

FIG. 18. Limit point for parameter $p_{14}$ on a path of fixed points of the annual map for $C_{l a b}$ for DALEC DE. The top branch is stable, the bottom branch is unstable and there is also a stable fixed point branch at $C_{l a b}=0$. The fixed points are sampled on day 0 of each year, which is December $21^{\text {st }}$.

consequence of this decoupling is that measurements of LAI can only constrain parameters in the foliar carbon pool. If the parameters in GPP and the parameter relating LAI to foliar carbon are kept fixed, as in REFLEX, then measurements of LAI can only hope to enable the fitting of the parameters $p_{5}, p_{11}$ and the combination $p_{3}\left(1-p_{2}\right)$. In DALEC EV, the fact that $p_{2}$ and $p_{3}$ are linked to different physiological mechanisms is irrelevant as far as the foliar carbon pool is concerned. This is an example of a general point that the sensitivity of the observables to the parameters in the model has a significant impact on whether it is possible to retrieve the values of the parameters from data. For example, in the DALEC model LAI is insensitive to $\tilde{p}_{4}$, so no data assimilation scheme will be able to retrieve the parameter $\tilde{p}_{4}$ from LAI alone. Furthermore, even if the observables are sensitive to the parameters, it may not be possible to identify all the parameters reliably (here, LAI is sensitive to both $p_{2}$ and to $p_{3}$, but it would only be possible to retrieve the combination $p_{3}\left(1-p_{2}\right)$ ).

With REFLEX, the participants had not just LAI data but also NEE data to try to 
constrain the parameters. However, it was still not possible to retrieve all parameter values reliably. Within carbon cycle models the multiple timescales, as illustrated in Fig. 15, means that it is inevitable that some model parameters are hard to extract from data and this issue will be investigated, in the context of DALEC, elsewhere.

We found that an increase in the atmospheric carbon parameter $C_{a}$ increases the region of the parameter plane where the forest will live, and also results in a higher value of $C_{f}$ at the stable non-trivial fixed point. Such a response is in agreement with ${ }^{31}$ who identify increased atmospheric carbon as a possible factor in increased growth of trees through carbon fertilisation. However, growth is also dependent on other resources, such as nutrients and stressors. ${ }^{32}$ found that during a six year experiment at a Free Air $\mathrm{CO}_{2}$ Enrichment (FACE) site in Duke Forest, USA, pines receiving elevated $\mathrm{CO}_{2}$ had on average about $17 \%$ more needles than untreated pines, mainly depending on the amount of nitrogen present in the soil.

Additionally we have shown that it may not be necessary in DALEC, for a forest that is living in a steady climate, to use daily driver data, but that it is sufficient to represent the drivers by a smooth cosine wave, where all that is needed is to measure the amplitude, mean and phase of the annual cycle. Clearly, smoothing the drivers would not always be appropriate, in particular when the model is used to study certain shock phenomena. We also acknowledge that smoothing the drivers takes away the natural variability, to which ecosystems respond. According to ${ }^{27}$, removal of high-frequency variability significantly enhances decadal Net Ecosystem Production, NEP (measurement of the net gain or loss of carbon in a system over a period of time), GPP and total respiration. Interestingly, they also show that solar radiation has a strong effect, whereas temperature variability only has a minor impact by comparison. During our smoothing exercise, we found that the model was more sensitive to solar radiation, which is why we had to resort to data assimilation methods to make the function fit, than to the temperature data. In DALEC EV, using the smoothed drivers did not make much difference to the results, perhaps as the high frequency variability was already taken out, the data being daily averages rather than hourly observations.

According to its creator, the DALEC EV model does seem to catch the qualitative behaviour of describing carbon stocks and stores: "We have attempted to strike the correct balance between sufficient model complexity to capture the essential dynamics of the system while maintaining simplicity" 19 and in future research it would be interesting to compare this 
simple model to more complex models. Photosynthesis sits at the heart of all process-based models and it is the nonlinearity of the photosynthesis model that underlies the structure that is seen in DALEC EV and DALEC DE so, even in more complex models, one should expect a similar bifurcation structure to occur. While it would be more challenging to use continuation methods on DGVMs that are more complex than DALEC, it may still be feasible. The same basic methods could be used: if climate drivers that are periodic on an annual scale are used then periodic solutions will exist that can be found by finding fixed points of an annual map. How these fixed points vary with parameters and their stability could also be investigated. Of course, climate drivers are not periodic, but as long as they are approximately periodic then one expects approximately periodic solutions. Bifurcation techniques that enable the computation of bifurcation structures even in noisy systems ${ }^{33}$ have been developed.

To conclude, an understanding of what sits at the heart of complex models, which a generic model such as DALEC represents, can give insight into parameter constrainability and have an impact on the choice of data assimilation schemes used.

\section{ACKNOWLEDGEMENTS}

AC gratefully acknowledges support from NERC via a studentship funded under the auspices of the National Centre for Earth Observation. We are grateful to Mathew Williams, University of Edinburgh, Timothy Hill, University of St Andrews, Shaun Quegan, University of Sheffield and Tristan Quaife, University of Reading, for helpful discussions regarding this work.

We are grateful to Shaun Quegan for the possible explanation for the parameter values for DALEC EV lying close to the line of limit points.

\section{Appendix A}

This appendix contains a description of the parameters in the DALEC models and their values.

\section{Parameters to be calibrated:}


Parameters used in DALEC EV

Description

Value

$p_{1}$ Daily decomposition rate

0.0000044100

$p_{2}$ Fraction of GPP respired

0.52

$p_{3}$ Fraction of NPP allocated to foliage

0.29

$\tilde{p}_{4}$ Fraction of NPP allocated to roots

0.2911

$p_{5}$ Daily turnover rate of foliage

0.0028

$p_{6}$ Daily turnover rate of wood

0.00000206

$p_{7}$ Daily turnover rate of roots

0.003

$p_{8}$ Daily mineralisation rate of litter

0.02

$p_{9}$ Daily mineralisation rate of soil and organic matter 0.00000265

$p_{10}$ Parameter in exponential term of temperature

dependent parameter

0.0693

$p_{11}$ Nitrogen use efficiency parameter in ACM

7.4 


\section{Parameters used in DALEC DE}

Description

Value

$p_{1}$ Daily decomposition rate 0.00001

$p_{2}$ Fraction of GPP respired 0.45

$p_{3}$ Fraction of NPP allocated to foliage $\quad 0.4$

$p_{4}$ Fraction of NPP remaining after allocation to foliage allocated to roots 0.4

$p_{5}$ Daily turnover rate of foliage $\quad 0.06$

$p_{6}$ Daily turnover rate of wood 0.00007

$p_{7}$ Daily turnover rate of roots 0.008

$p_{8}$ Daily mineralisation rate of litter 0.03

$p_{9}$ Daily mineralisation rate of soil and organic matter 0.00003

$p_{10}$ Parameter in exponential term of temperature dependent parameter 0.073

$p_{11}$ Nitrogen use efficiency parameter in ACM 14

$p_{12}$ GDD value causing leaf out (GDD is the growing degree day factor) 240

$p_{13}$ Minimum daily temperature causing leaf fall $\quad 9$

$p_{14}$ Fraction of leaf loss transferred to litter 0.48

$p_{15}$ Daily turnover rate of labile carbon 0.09

$p_{16}$ Fraction of labile transfers respired 0.15

$p_{17}$ Maximum $C_{f}$ value $\left(\mathrm{g} \mathrm{C} \mathrm{m}^{-2}\right) \quad 300$ 
Parameters viewed as constants, all in the ACM:

\section{Optimised Value}
$a_{2} \quad 0.0156$
$a_{3} \quad 4.22273$
$a_{4} \quad 208.868$
$a_{5} \quad 0.0453$
$a_{6} \quad 0.3783$
$a_{7} \quad 7.1929$
$a_{8} \quad 0.0111$
$a_{9} \quad 2.1001$
$a_{10} 0.7897$

\section{Appendix B}

This appendix contains the functions in the GPP. Recall that the GPP, as defined in (12), is

$$
G P P\left(C_{f}(t), t\right)=\frac{E_{0}\left(C_{f}(t)\right) I(t) g_{c}(t)\left(C_{a}(t)-C_{i}\left(C_{f}(t), t\right)\right)}{E_{0}\left(C_{f}(t)\right) I(t)+g_{c}(t)\left(C_{a}(t)-C_{i}\left(C_{f}(t), t\right)\right)}\left(a_{2} s(t)+a_{5}\right) .
$$

Canopy Conductance, $\mathrm{g}_{\mathrm{c}}(t)$

$$
g_{c}(t)=\frac{|\psi|^{a_{10}}}{0.5 T_{r}(t)+a_{6} R_{t o t}}
$$

where:

$\psi \quad=2$ (constant),
$R_{\text {tot }}=1$ (constant).

$\mathrm{CO}_{2}$ Concentration at Site of Carboxylation, $\mathrm{C}_{\mathrm{i}}\left(\mathrm{C}_{\mathrm{f}}(\mathrm{t}), \mathrm{t}\right)$

$C_{i}\left(C_{f}(t), t\right)=\frac{1}{2}\left[C_{a}+q-p\left(C_{f}(t), t\right)+\sqrt{\left(C_{a}+q-p\left(C_{f}(t), t\right)\right)^{2}-4\left(C_{a} q-a_{3} p\left(C_{f}(t), t\right)\right)}\right]$, 
where:

$C_{a}=380$ (constant averaged over three years);

$q \quad=a_{3}-a_{4}=-204.64527$

Photosynthate, $\mathrm{p}\left(\mathrm{C}_{\mathrm{f}}(\mathrm{t}), \mathrm{t}\right)$

$$
p\left(C_{f}(t), t\right)=\frac{p_{11} N C_{f}(t)}{\operatorname{lma} g_{c}(t)} \exp \left(a_{8} T_{\max }(t)\right)
$$

where:

$N=4(\mathrm{EV})$ and $2.7(\mathrm{DE})$ (constant),

$\operatorname{lma}=110(\mathrm{EV}), 60(\mathrm{DE})$ (constant).

Canopy Level Quantum Yield, $\mathrm{E}_{0}\left(\mathrm{C}_{\mathrm{f}}(\mathrm{t})\right)$

$$
E_{0}\left(C_{f}(t)\right)=\frac{a_{7} C_{f}(t)^{2}}{C_{f}(t)^{2}+a_{9} \operatorname{lma}^{2}}
$$

where:

$\operatorname{lma}=110(\mathrm{EV}), 60(\mathrm{DE})($ constant $)$.

Solar Declination

$$
\delta(t)=-23.4 \cos (2 \pi t / 365)(\pi / 180)=-0.408 \cos (2 \pi t / 365) .
$$

Day Length

$$
s(t)=24 \cos ^{-1}(-\tan (\text { lat }) \tan (\delta(t))) / \pi,
$$

where:

lat $=52^{\circ}(\mathrm{EV}$, constant $), 42.5^{\circ}(\mathrm{DE}$, constant $)$ 


\section{Appendix C}

This appendix contains a list of symbols.

\section{Symbol Description}

$\psi \quad$ Max soil-leaf water potential difference (MPa)

$T_{r} \quad$ Daily temperature range $\left({ }^{\circ} \mathrm{C}\right)$

$R_{t o t} \quad$ Total plant-soil hydraulic resistance $\left(\mathrm{MPa} \mathrm{m}{ }^{2} \mathrm{~s} \mathrm{mmol}^{-1}\right)$

$N \quad$ Foliar nitrogen $\left(\mathrm{g} \mathrm{N} \mathrm{m}^{-2}\right.$ leaf area)

lma Leaf mass per area $\left(\mathrm{g} \mathrm{C} \mathrm{m}^{-2}\right.$ leaf area)

$L \quad$ Leaf area index $\left(\mathrm{m}^{2} \mathrm{~m}^{-2}\right)(\mathrm{LAI})$

$T_{\max } \quad$ Maximum daily temperature $\left({ }^{\circ} \mathrm{C}\right)$

$T_{m} \quad$ Mean daily air temperature $\left({ }^{\circ} \mathrm{C}\right)$

$C_{a} \quad$ Atmospheric $\mathrm{CO}_{2}$ concentration $\left(\mu \mathrm{mol} \mathrm{mol}{ }^{-1}\right)$

$\delta \quad$ Solar declination (radians)

$D \quad$ Day of the year

lat $\quad$ Site latitude $\left(^{\circ}\right)$

$I \quad$ Irradiance $\left(\mathrm{MJ}^{-1} \mathrm{~m}^{-2}\right.$ day $\left.{ }^{-1}\right)$

$R_{a} \quad$ Autotrophic respiration

$R_{h} \quad$ Heterotrophic respiration, $R_{h_{1}}+R_{h_{2}}\left(\mathrm{~g} \mathrm{C} \mathrm{m}^{-2}\right)$

$$
R_{h_{1}}: p_{8} C_{l i t} T(t)
$$

$R_{h_{2}}: p_{9} C_{\text {som }} T(t)$

\section{Appendix D}

This appendix contains the parameter and other constant values for the Oregon forest.

Parameters viewed as constants, all in ACM: 


\section{Optimised Value}

$a_{2} \quad 0.0142$

$a_{3} \quad 0.980$

$a_{4} \quad 217.9$

$a_{5} \quad 0.155$

$a_{6} \quad 2.653$

$a_{7} \quad 4.309$

$a_{8} \quad 0.060$

$a_{9} 1.062$

$a_{10} 0.0006$

Parameters to be calibrated:

\section{Description}

$p_{1}$ Daily decomposition rate

$p_{2}$ Fraction of GPP respired

$p_{3}$ Fraction of NPP allocated to foliage

$\tilde{p}_{4}$ Fraction of NPP allocated to roots

$p_{5}$ Daily turnover rate of foliage

$p_{6}$ Daily turnover rate of wood

$p_{7}$ Daily turnover rate of roots

$p_{8}$ Daily mineralisation rate of litter

$p_{9}$ Daily mineralisation rate of soil and organic matter 0.00000265

$p_{10}$ Parameter in exponential term of temperature dependent parameter

$p_{11}$ Nitrogen use efficiency parameter in ACM
Value in our analysis

0.0000044100

0.473267

0.314951

0.297586

0.00266518

0.00000206

0.00248

0.028

0.0693

2.155

Other: 
Value

$\psi \quad-0.8502$

$R_{\text {tot }} 1$

N 2.7

lat $52^{\circ}$

lma 111

$C_{a} 357$ (averaged over three years of data) 


\section{REFERENCES}

${ }^{1}$ J.A. Foley et al., "Green surprise? How terrestrial ecosystems could affect Earth's climate," Frontiers in Ecology and the Environment 1, 38-44 (2003).

${ }^{2}$ W. Knorr and M. Heimann, "Uncertainties in global terrestrial biosphere modeling, Part I: A comprehensive sensitivity analysis with a new photosynthesis and energy balance scheme," Global Biogeochemical Cycles 15, 207-225 (March 2001).

${ }^{3}$ P.M. Cox, "Description of the TRIFFID dynamic global vegetation model," Tech. Rep. 24 (Hadley Centre, 2001).

${ }^{4}$ S. Sitch et al., "Evaluation of ecosystem dynamics, plant geography and terrestrial carbon cycling in the LPJ dynamic global vegetation model," Global Change Biology 9, 161-185 (2003).

${ }^{5}$ H. Sato, A. Itoh, and T. Kohyama, "SEIB-DGVM: A new dynamic global vegetation model using a spatially explicit individual-based approach," Ecological Modelling 200, 279-307 (2007).

${ }^{6}$ D. Medvigy et al., "Mechanistic scaling of ecosystem function and dynamics in space and time: Ecosystem Demography model version 2.." Journal of Geophysical Research 114, GO1002, doi: 10.1029/2008JG000812 (2009).

${ }^{7}$ A.D. Friend et al., "A process-based, terrestrial biosphere model of ecosystem dynamics (Hybrid v3.0)," Ecological Modelling 95, 248-287 (1997).

${ }^{8}$ F.I. Woodward, T.M. Smith, and W.R. Emmanuel, "A global land primary productivity and phytogeography model," Global Biogeochemical Cycles 9, 471-490 (1995).

${ }^{9}$ M. Heimann, "The global atmospheric tracer model tm2 technical report no. 10.." MaxPlanck-Institute for Meteorology, Hamburg, Germany(1995).

${ }^{10}$ M.J. Best et al., "The joint UK land environment simulator (JULES), model description - Part 1: Energy and water fluxes," Geoscientific Model Development 4, 677-699 (2011).

${ }^{11} \mathrm{~S}$. Schaphoff et al., "Terrestrial biosphere carbon storage under alternative climate projections," Climatic Change 74, 97-122 (2006).

${ }^{12}$ M. Maslin and P. Austin, "Climate models at their limit?." Nature 486, 183-184 (2012).

${ }^{13} \mathrm{~T}$. Ziehn et al., "Improving the predictability of global co2 assimilation rates under climate change," Geophysical Research Letters 38, L10404 doi:10.1029/2011GL047182 (2011).

${ }^{14}$ T. Hill, E. Ryan, and M. Williams, "The use of $\mathrm{CO}_{2}$ flux time series for parameter and 
carbon stock estimation in carbon cycle research," Global Change Biology 18, 179-193 (2012).

${ }^{15}$ E.N. Lorenz, "Deterministic nonperiodic flow," Journal of the Atmospheric Sciences 20, 130-141 (1963).

${ }^{16} \mathrm{M}$. Williams et al., "An improved analysis of forest carbon dynamics using data assimilation," Global Change Biology 11, 89-105 (2005).

${ }^{17}$ G.D. Farquhar and S. von Caemmerer, "A biochemical model of photosynthetic $\mathrm{CO}_{2}$ assimilation in leaves of C3 species.." Planta 149, 78-90 (1980).

${ }^{18} \mathrm{~T}$. Ilett, An analysis of a discrete-time nonlinear dynamical system, Master's thesis, University of York (2008).

${ }^{19}$ A. Fox et al., "The REFLEX project: Comparing different algorithms and implementations for the inversion of a terrestrial ecosystem model against eddy covariance data," Agricultural and Forest Meteorology 149, 1597-1615 (2009).

20 "Reflex experiment document, http://www.geos.ed.ac.uk/carbonfusion/events/reflex.html," (2007).

${ }^{21} \mathrm{M}$. Williams et al., "Predicting gross primary productivity in terrestrial ecosystems," Ecological Applications 7, 882-894 (1997).

${ }^{22} \mathrm{M}$. Williams et al., "Modelling the soil-plant-atmosphere continuum in a Quercus-Acer stand at Harvest Forest: the regulation of stomatal conductance by light, nitrogen and soil/plan hydraulic properties," Plant, Cell and Environment 19, 911-927 (1996).

${ }^{23}$ H.G. Jones, Plants and microclimate: a quantitive approach to environmental plant physiology, 2nd ed. (Cambridge University Press, 1992).

${ }^{24}$ P. Foukal, C. Fröhlich, H. Spruit, and T. M. L. Wigley, "Variations in solar luminosity and their effect on the earth's climate," Nature 443, 161-166 (2006).

${ }^{25}$ A. Dhooge, W. Govaerts, and Yu. A. Kuznetsov, "Matcont: A matlab package for numerical bifurcation analysis of odes.." ACM Transactions on Mathematical Software (TOMS) TOMS Homepage table of contents archive 29, 141-164 (June 2003).

${ }^{26} \mathrm{O}$. Talagrand and P. Courtier, "Variational assimilation of meteorological observations with the adjoint vorticity equation. i: Theory," Quart. J. Roy. Met. Soc. 113, 1311-1328 (1987).

${ }^{27} \mathrm{D}$. Medvigy et al., "Responses of terrestrial ecosystems and carbon budgets to current and future environmental variability.." Proceedings of the National Academy of Sciences 107, 
$8275-8280(2010)$.

${ }^{28}$ J.G. Cannadell et al., "Contributions to accelerating atmospheric $\mathrm{CO}_{2}$ growth from economic activity, carbon intensity and efficiency of natural sinks," Proceedings of the National Academy of Sciences 104, 18866-18870 (2007).

${ }^{29}$ C. Greene, B. Monger, and M. Huntley, "Geo-engineering: The inescapable truth of getting to 350 ," Solutions 1, 57-66 (2010).

${ }^{30}$ P.F. Bordeau, "Seasonal variations of the photosynthetic efficiency of evergreen conifers," Ecology 40, 63-67 (1959).

${ }^{31}$ S.M. McMahon, G.G. Parker, and D.R. Millera, "Evidence for a recent increase in forest growth," Proceedings of the National Academy of Sciences 107, 3611-3615 (2010).

${ }^{32} \mathrm{H}$. McCarthy et al., "Temporal dynamics and spacial variability in the enhancement of canopy leaf area under elevated atmospheric $\mathrm{CO}_{2}$," Global Change Biology 13, 2479-2497 (2007).

${ }^{33}$ D. Barkley, I. G. Kevrekidis, and A. M. Stuart, "The moment map: Nonlinear dynamics of density evolution via a few moments," SIAM Journal of Applied Dynamical Systems 5, 403-434 (2006). 